TRANSACTIONS OF THE

AMERICAN MATHEMATICAL SOCIETY

Volume 365, Number 5, May 2013, Pages 2779-2802

S 0002-9947(2012)05719-9

Article electronically published on November 26, 2012

\title{
DIAMETER OF GRAPHS OF REDUCED WORDS AND GALLERIES
}

\author{
VICTOR REINER AND YUVAL ROICHMAN
}

Abstract. For finite reflection groups of types $A$ and $B$, we determine the diameter of the graph whose vertices are reduced words for the longest element and whose edges are braid relations. This is deduced from a more general theorem that applies to supersolvable hyperplane arrangements.

\section{INTRODUCTION}

The symmetric group $W=\mathfrak{S}_{n}$ on $n$ letters has a well-known Coxeter presentation, with generating set $S=\left\{s_{1}, \ldots, s_{n-1}\right\}$ consisting of the adjacent transpositions $s_{i}=(i, i+1)$, satisfying the braid relations

$$
\text { (ii) } s_{i} s_{i+1} s_{i}=s_{i+1} s_{i} s_{i+1} \quad \text { for } 1 \leq i \leq n-2 \text {, }
$$

together with the condition that each $s_{i}$ is an involution. Given any $w$ in $W$, a reduced decomposition for $w$ is a sequence $\left(s_{i_{1}}, \ldots, s_{i_{\ell}}\right)$ of the generators $S$ for which $w=s_{i_{1}} \cdots s_{i_{\ell}}$ that attains the minimum possible length $\ell=: \ell(w)$.

There is a well-studied graph $G(w)$ whose vertex set is the set $\mathcal{R}(w)$ of all reduced decompositions of $w$, and whose edges correspond to the applicable braid relations (i) and (ii) above. A theorem of Tits [30, (see also [10, Theorem 3.3.(ii)]) says that for any finite Coxeter group $(W, S)$ and any $w$ in $W$, this graph $G(w)$ is connected. A particularly interesting special case occurs when $w$ is the unique longest element $w_{0}$ of $W$. For $W=\mathfrak{S}_{4}$, the graph $G\left(w_{0}\right)$ is illustrated in Figure 1.1, where each reduced word is abbreviated by its subscript sequence (e.g., 121321 for $\left.\left(s_{1}, s_{2}, s_{1}, s_{3}, s_{2}, s_{1}\right)\right)$, and with braid relations of type (i) darkened.

The graph $G\left(w_{0}\right)$ and some of its generalizations were shown to have further graph-theoretic connectivity in work of Athanasiadis, Edelman and Reiner [3], and Athanasiadis and Santos [2]. This was motivated by earlier topological connectivity results surrounding a closely related poset, appearing first in a conjecture of Baues [6] on loop spaces, which was proven in work of Billera, Kapranov, and Sturmfels [7] and Björner 9]. We also mention here a few ancillary results about the graph $G\left(w_{0}\right)$. Tits [31] gave explicit generators for its fundamental group. Stanley 28 was the first to show that its vertex set $\mathcal{R}\left(w_{0}\right)$ is equinumerous with the standard Young tableaux of shape $(n-1, n-2, \ldots, 2,1)$. In $[23$, the average degree of a vertex of $G\left(w_{0}\right)$ with respect to only the edges of type (ii) was shown to

Received by the editors June 28, 2011 and, in revised form, October 5, 2011.

2010 Mathematics Subject Classification. Primary 20F55, $20 \mathrm{~F} 05$.

Key words and phrases. Coxeter group, reduced words, supersolvable, hyperplane arrangement, weak order, reflection order, cellular string, zonotope, monotone path, diameter.

The first author was supported by NSF grant DMS-0245379.

The second author was supported in part by the Israel Science Foundation grant \# 947/04. 


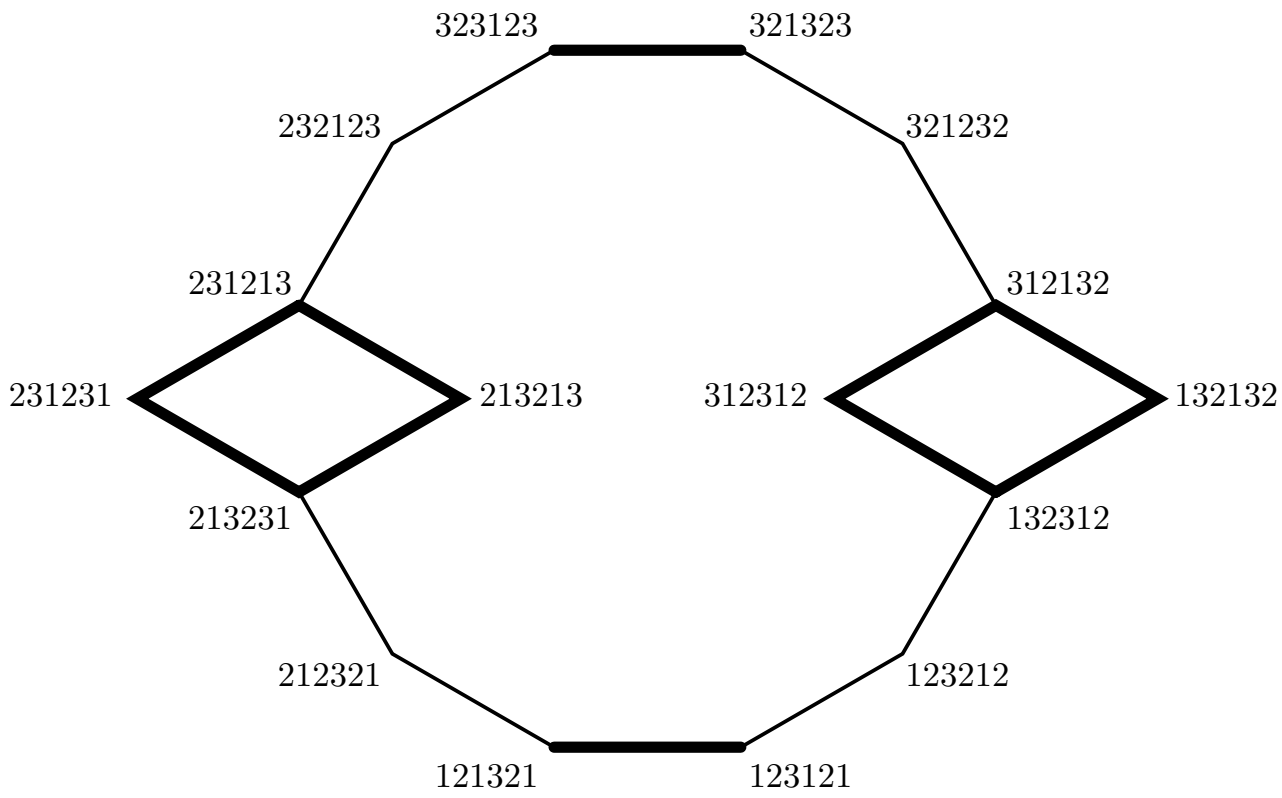

Figure 1.1. The graph $G\left(w_{0}\right)$ for $W=\mathfrak{S}_{4}$.

be 1. Manin and Schechtman [19, Ziegler [32, Felsner [17, and Shapiro, Shapiro and Vainshtein 26] have studied, in the guise of the higher Bruhat order B(n,2), the quotient graph of $G\left(w_{0}\right)$ in which one contracts down all its edges of type (i).

However, the diameter of $G\left(w_{0}\right)$, seems to have been considered only very recently. Autord and Dehornoy [4, Proposition 1] show that for $W=\mathfrak{S}_{n}$, the diameter of $G\left(w_{0}\right)$ grows asymptotically in $n$ as a constant times $n^{4}$. Our main result, Theorem 1.1, shows the diameter is exactly $\frac{1}{24}(n-2)(n-1) n(3 n-5)$, which is the number of codimension-two subspaces arising as intersections of two hyperplanes $x_{i}=x_{j}$ in the reflection arrangement associated to $W=\mathfrak{S}_{n}$.

1.1. Diameter of $G\left(w_{0}\right)$ and supersolvable hyperplane arrangements. The graph $G\left(w_{0}\right)$ for any finite Coxeter group $(W, S)$ has a natural generalization to the context of real hyperplane arrangements that first arose in work of Deligne 15, and later Salvetti [25, on the topology of the complexified complements of these arrangements. This was generalized further to the context of oriented matroids by Cordovil and Moreira [14; to decrease technicalities and enhance readability, we will mainly adhere to the language of hyperplane arrangements in this paper. We review the arrangement viewpoint here in order to state Theorem 1.1, see [12, $\S 4.4$, pp. 184-186] and Remark 2.5 below for further discussion.

Let $\mathcal{A}$ be an arrangement of finitely many linear hyperplanes in $\mathbb{R}^{d}$ that is central and essential, meaning that $\bigcap_{H \in \mathcal{A}} H=\{0\}$. Let $L=\bigsqcup_{i=0}^{d} L_{i}$ be its graded poset of intersection subspaces, ordered via reverse inclusion. 
Define a graph structure $G_{1}$ on the set $\mathcal{C}$ of chambers of $\mathcal{A}$, in which two chambers $c, c^{\prime}$ are connected by an edge when they are separated by exactly one hyperplane $H$ in $L_{1}$. It is well known, and will be recalled in Section 3 , why this graph $G_{1}$ always has diameter exactly $\left|L_{1}\right|=|\mathcal{A}|$, that is, the number of hyperplanes.

Now choose a particular base chamber $c_{0}$, and let $\mathcal{R}$ denote the set of all minimal galleries $r$ (that is, geodesics in $G_{1}$ ) from $c_{0}$ to $-c_{0}$. There is a graph structure $G_{2}$ on this set $\mathcal{R}$, in which two galleries $r, r^{\prime}$ are connected by an edge when they are separated (in a sense made precise in Section 21) by exactly one codimensiontwo intersection subspace $X$ in $L_{2}$. This graph $G_{2}$ is known to be connected (see Remark 2.5 below), and it will be shown in Section 3 that its diameter is always at least $\left|L_{2}\right|$, raising the following question.

Main question. For real hyperplane arrangements $\mathcal{A}$ and a choice of base chamber $c_{0}$, does the graph $G_{2}$ of minimal galleries from $c_{0}$ to $-c_{0}$ have diameter $\left|L_{2}\right|$ ?

Remark 2.6] discusses why the answer to this question is affirmative for hyperplane arrangements in dimension at most 3 , and even for oriented matroids of rank at most 3 , by a result of Cordovil, but negative for oriented matroids in rank 4 , by an example of Richter-Gebert. After acceptance of this paper for publication, computations by Rob Edman provided a negative answer for the 4-dimensional cyclic arrangement of 8 hyperplanes, that is, the hyperplanes in $\mathbb{R}^{4}$ normal to the vectors $v_{i}:=\left(1, t_{i}, t_{i}^{2}, t_{i}^{3}\right)$ for real numbers $t_{1}<t_{2}<\cdots<t_{8}$. Specifically, in this example, one finds several choices of base chamber $c_{0}$ (including the chamber $c_{0}$ where vectors have positive dot product with all 8 of the $v_{i}$ ) for which the graph $G_{2}$ of minimal galleries from $c_{0}$ to $-c_{0}$ has diameter $30>28=\left(\begin{array}{l}8 \\ 2\end{array}\right)=\left|L_{2}\right|$.

Note that when $\mathcal{A}$ is the arrangement of reflecting hyperplanes for a finite real reflection group $W$, the choice of base chamber $c_{0}$ is immaterial, as $W$ acts simply transitively on the chambers $\mathcal{C}$. Also, in this case the graph $G_{2}$ is easily seen to be exactly the graph of reduced words for the longest element $w_{0}$ in $W$ described above.

The following main result answers the main question affirmatively for reflection arrangements of types $A, B$, as well as the more general supersolvable arrangements. See Section 4 for undefined terms in its statement.

Theorem 1.1. When $\mathcal{A}$ is a supersolvable hyperplane arrangement, and the base chamber $c_{0}$ is chosen incident to a modular flag, the graph $G_{2}$ has diameter $\left|L_{2}\right|$.

In particular, for the reflection arrangements of type $A_{n-1}$ and $B_{n}$ and the dihedral groups $I_{2}(m)$, the graphs of reduced words for $w_{0}$ have diameters given by the values of $\left|L_{2}\right|$ shown in Table 1.1 .

Unfortunately, types $A_{n-1}, B_{n}$, and $I_{2}(m)$ are the only irreducible real reflection group $\$ 1$ whose reflection arrangements are supersolvable; see Barcelo and Ihrig 5 , Theorem 5.1].

We remark here on some of the data related to Table1.1. For type $D_{4}$, computer calculation 2 by Rob Edman show that one has $\left|\mathcal{R}\left(w_{0}\right)\right|=2316$, in agreement with [28, $\S 7]$. In addition, these calculations exhibit many reduced words for $w_{0}$ giving rise to nodes of the graph $G_{2}$ which are $L_{2}$-accessible in the sense of Definition 3.9

\footnotetext{
${ }^{1}$ Note that taking products of hyperplane arrangements [20, Def. 2.13] which are supersolvable preserves supersolvability, and every finite real reflection group has its reflection arrangement equal to a product of reflection arrangements for irreducible real reflection groups.

${ }^{2}$ These computations give $\left|\mathcal{R}\left(w_{0}\right)\right|$ in type $D_{5}$ as 12985968 , and in type $D_{6}$ as 3705762080 .
} 
TABLE 1.1

\begin{tabular}{|c|c|c|}
\hline$W$ & $\left|L_{2}\right|$ & Does $G_{2}$ have diameter $\left|L_{2}\right| ?$ \\
\hline \hline$A_{n-1}$ & $\frac{1}{24} n(n-1)(n-2)(3 n-5)$ & Yes, by Theorem 1.1 \\
\hline$B_{n}$ & $\frac{1}{6} n(n-1)\left(3 n^{2}-5 n+1\right)$ & Yes, by Theorem 1.1 \\
\hline$D_{n}$ & $\frac{1}{6} n(n-1)\left(3 n^{2}-11 n+13\right)$ & Yes, for $n \leq 4 ;$ unknown generally. \\
\hline$E_{8}$ & 4900 & Unknown. \\
\hline$E_{7}$ & 1281 & Unknown. \\
\hline$E_{6}$ & 390 & Unknown. \\
\hline$F_{4}$ & 122 & Unknown. \\
\hline$H_{4}$ & 722 & Unknown. \\
\hline$H_{3}$ & 31 & Yes, by Theorem 2.7 \\
\hline$I_{2}(m)$ & 1 & Yes, trivially. \\
\hline
\end{tabular}

and Proposition 3.12 below, showing that the diameter is $\left|L_{2}\right|$. Interestingly, none of the $L_{2}$-accessible nodes come from reduced words that are lexicographically first among all reduced words, no matter how one linearly orders the Coxeter generators. For $F_{4}$, these calculations show that $\left|\mathcal{R}\left(w_{0}\right)\right|=2144892$, making the full diameter calculation harder, but again, the computer has checked that none of the lexicographically first reduced words gives an $L_{2}$-accessible node.

The remainder of the paper is structured as follows. Section 2 establishes formal definitions for the graph $G_{2}$ to be studied, remarking on its connectivity, its diameter in low dimension, as well as its relation to mononotone path zonotopes. Section 3 introduces the notion of a set-valued metric on a graph, which is then applied in Section 4 to prove Theorem 1.1. Section 5 explains how some of these results adapt for the graphs $G(w)$ when $w$ is not the longest element $w_{0}$.

\section{Arrangements And the graphs $G_{1}, G_{2}$}

We review here some of the theory of hyperplane arrangements; see Orlik and Terao 20] and Stanley 29] for good references.

As in the Introduction, $\mathcal{A}=\left\{H_{1}, \ldots, H_{N}\right\}$ will be an arrangement of hyperplanes in $\mathbb{R}^{d}$, which is central and essential, that is, $\bigcap_{i=1}^{N} H_{i}=\{0\}$. The intersection poset $L$ for $\mathcal{A}$ is the collection of intersection subspaces $X=\bigcap_{i \in I} H_{i}$ of subsets of the hyperplanes, ordered by reverse inclusion. This makes $L$ a geometric lattice (see [20, §2.1]), and therefore graded or ranked. Let $L=\bigsqcup_{i=0}^{d} L_{i}$ be the decomposition into its ranks, so that

- the bottom rank $L_{0}$ contains only the empty intersection $\mathbb{R}^{d}$ itself,

- the set of atoms $L_{1}$ is the set of hyperplanes $\left\{H_{1}, \ldots, H_{n}\right\}=\mathcal{A}$, and

- the top rank $L_{d}$ contains only the zero subspace $\{0\}$.

The complement $\mathbb{R}^{d} \backslash \mathcal{A}$ decomposes into a collection $\mathcal{C}$ of connected components called chambers. Given two chambers $c, c^{\prime}$, define their separation set

$$
L_{1}\left(c, c^{\prime}\right):=\left\{H \in L_{1}=\mathcal{A}: H \text { separates } c \text { from } c^{\prime}\right\} .
$$




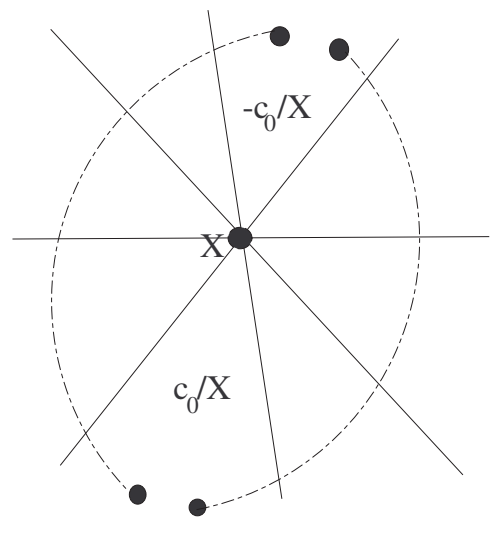

Figure 2.1. The two possibilities for a minimal gallery $r / X$ from $c_{0} / X$ to $-c_{0} / X$ through $\mathcal{A}_{X}$ when $X$ has codimension-two, each shown as a dashed path.

Definition 2.1 (The graph $G_{1}$ ). Given an arrangement $\mathcal{A}$, define a graph $G_{1}$ whose vertex set is the set of chambers $\mathcal{C}$, and having an edge between two chambers $\left\{c, c^{\prime}\right\}$ exactly when $\left|L_{1}\left(c, c^{\prime}\right)\right|=1$.

A (minimal) gallery from chamber $c$ to chamber $c^{\prime}$ is a geodesic (shortest path)

$$
c:=c_{0}, c_{1}, \ldots, c_{d-1}, c_{d}:=c^{\prime}
$$

in this graph $G_{1}$. Fixing one particular choice of a base chamber $c_{0}$, let $\mathcal{R}$ denote the set of all minimal galleries $r$ from $c_{0}$ to $-c_{0}$.

We wish to discuss how the codimension-two intersection subspaces in $L_{2}$ can separate minimal galleries. A minimal gallery $r$ in $\mathcal{R}$ must cross every hyperplane $H_{1}, \ldots, H_{N}$ of $\mathcal{A}$ exactly once, and is completely determined by the linear order in which they are crossed. Given any intersection subspace $X$, one defines the localized arrangement of hyperplanes in the quotient space $\mathbb{R}^{d} / X$

$$
\mathcal{A}_{X}:=\{H / X: H \in \mathcal{A} \text { and } H \supseteq X\} .
$$

Note that the intersection lattice for $\mathcal{A}_{X}$ may be identified with the lower interval $\left[\mathbb{R}^{d}, X\right]$ within $L$. For each chamber $c$ of $\mathcal{A}$, there is a unique chamber $c / X$ of $\mathcal{A}_{X}$ that contains all additive cosets of the subspace $X$ represented by points of $c$. A minimal gallery $r$ in $\mathcal{R}$ from $c_{0}$ to $-c_{0}$ induces a minimal gallery $r / X$ from $c_{0} / X$ to $-c_{0} / X$ in $\mathcal{A}_{X}$. In particular, when $X$ has codimension two, so that $\mathcal{A}_{X}$ is an arrangement of lines through the origin in the 2-dimensional plane $\mathbb{R}^{d} / X$, every minimal gallery $r$ has exactly two possibilities for the induced minimal gallery $r / X$ from $c_{0} / X$ to $-c_{0} / X$ in $\mathcal{A}_{X}$; see Figure 2.1.

Given two minimal galleries $r, r^{\prime}$ in $\mathcal{R}$ and a codimension-two subspace $X$ in $L_{2}$, say that $X$ separates $r$ from $r^{\prime}$ if $r / X \neq r^{\prime} / X$. Define their separation set

$$
L_{2}\left(r, r^{\prime}\right):=\left\{X \in L_{2}: X \text { separates } r \text { from } r^{\prime}\right\} .
$$

Definition 2.2 (The graph $G_{2}$ ). Given the arrangement $\mathcal{A}$ and the chosen base chamber $c_{0}$ in $\mathcal{C}$, define a graph $G_{2}$ whose vertex set is the set $\mathcal{R}$ of minimal galleries $c_{0}$ to $-c_{0}$, and having an edge between two galleries $\left\{r, r^{\prime}\right\}$ exactly when $\left|L_{2}\left(r, r^{\prime}\right)\right|=1$. 


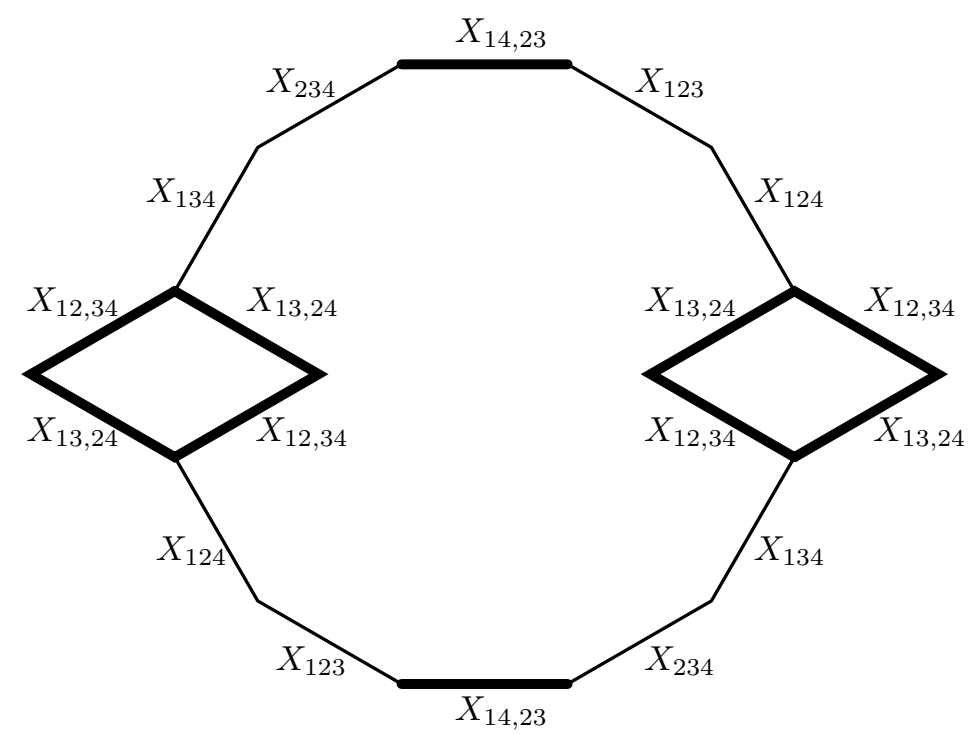

Figure 2.2. Labelling the edges $\left\{r, r^{\prime}\right\}$ in $G\left(w_{0}\right)$ for $w_{0}$ by the unique element $X$ in $L_{2}\left(r, r^{\prime}\right)$

The following proposition points out how separation sets $L_{i}(-,-)$ for $i=1,2$ encode the chambers $\mathcal{C}$ and galleries $\mathcal{R}$.

Proposition 2.3. Given any fixed base chamber $c_{0}$, the set $L_{1}\left(c_{0}, c\right)$ determines the chamber $c$ uniquely. Given any fixed base gallery $r_{0} \in \mathcal{R}$ from $c_{0}$ to $-c_{0}$, the set $L_{2}\left(r_{0}, r\right)$ determines the gallery $r$ uniquely.

Proof. The first assertion is clear (and implicit in the discussion of [16, §I]) since $L_{1}\left(c_{0}, c\right)$ determines on which side of each hyperplane $H$ of $\mathcal{A}$ the chamber $c$ lies.

For the second assertion, as noted earlier, since $L_{1}\left(c_{0},-c_{0}\right)=L_{1}=\mathcal{A}$, the gallery $r$ from $c_{0}$ to $-c_{0}$ must cross every hyperplane $H$ of $\mathcal{A}$, and $r$ is determined by the linear order in which it crosses these hyperplanes. This linear order is determined by knowing for each pair $H, H^{\prime}$ which of the two is crossed first. The latter is determined from the order in which $r$ crosses the hyperplanes of the localized arrangement $\mathcal{A}_{X}$ for the codimension-two subspace $X:=H \cap H^{\prime}$, and this is encoded by the separation set $L_{2}\left(r_{0}, r\right)$.

Example 2.4. The reflection arrangement of type $A_{n-1}$, corresponding to the symmetric group $W=\mathfrak{S}_{n}$, has ambient space isomorphic to $\mathbb{R}^{d}$ for $d=n-1$; one identifies $\mathbb{R}^{n-1}$ with the quotient of $\mathbb{R}^{n}$ (having coordinates $x_{1}, \ldots, x_{n}$ ) by the subspace $x_{1}=x_{2}=\cdots=x_{n}$. Its hyperplanes are $H_{i j}:=\left\{x_{i}=x_{j}\right\}$ for $1 \leq i<j \leq n$, and its codimension-two intersection subspaces in $L_{2}$ are either of type (i) $X_{i j, k \ell}:=\left\{x_{i}=x_{j}, x_{k}=x_{\ell}\right\}$ or of type (ii) $X_{i j k}:=\left\{x_{i}=x_{j}=x_{k}\right\}$, corresponding to the braid relations of types (i), (ii) from the Introduction. The 
graph $G\left(w_{0}\right)$ from (1.1) is redrawn in Figure 2.2, with each edge $\left\{r, r^{\prime}\right\}$ labeled by the unique codimension-two subspace $X$ separating $r$ from $r^{\prime}$.

We close this section with three remarks on the graph $G_{2}$. All of these can be safely skipped by the reader solely interested in the proof of Theorem 1.1 .

Remark 2.5 (On the connectivity of $G_{2}$ ). It is not obvious that the graph $G_{2}$ is connected for every real hyperplane arrangement $\mathcal{A}$ and every choice of base chamber $c_{0}$. However, as mentioned in the Introduction, this connectivity of $G_{2}$ was proven at the following successively stronger levels of generality:

- for real reflection arrangements by Tits 30,

- for real simplicial arrangements by Deligne [15],

- for all real arrangements by Salvetti [25], and

- for oriented matroids by Cordovil and Moreira [14].

Remark 2.6 (On the diameter of $G_{2}$ in low dimension). When the arrangement $\mathcal{A}$ lives in $\mathbb{R}^{d}$ for $d \leq 2$, regardless of the choice of base chamber $c_{0}$, the graph $G_{2}$ is trivial, consisting of a single vertex for $d=1$, and consisting of two vertices connected by a single edge for $d=2$.

When $d=3$, regardless of the choice of base chamber $c_{0}$, the diameter of $G_{2}$ is exactly $\left|L_{2}\right|$ by the following result of Cordovil [14, Theorem 2.5] (cited there as being implicit in [13, Theorem 2.1]), and proven even more generally for rank 3 oriented matroids:

Theorem 2.7 (Cordovil). In a 3-dimensional real central hyperplane arrangement $\mathcal{A}$ (or even a rank 3 oriented matroid), any two minimal galleries $r, r^{\prime}$ from a chamber $c_{0}$ to its opposite $-c_{0}$ can be connected by a sequence of at most $\left|L_{2}(\mathcal{A})\right|$ elementary deformations.

When $d=4$, this assertion fails at the level of generality of oriented matroids; the authors thank Jim Lawrence for pointing out how this follows from an important counterexample of J. Richter-Gebert, which we recapitulate here; see [12] for most of the oriented matroid terminology left undefined.

A crucial notion is that of a strong map $N \rightarrow M$, where $N, M$ are oriented matroids on the same ground set $E$; this is defined [12, $\S 7.7]$ by requiring that the covectors of $M$, as a subset of $\{0,+,-\}^{E}$, form a subset of the covectors of $N$. This combinatorially abstracts the arrangement picture as follows. When $N$ comes from a collection of vectors $\left\{v_{e}\right\}_{e \in E}$ in $\mathbb{R}^{d}$, thought of as the normal vectors to the hyperplanes of $\mathcal{A}$, then its covectors are the sign vectors $\left(\operatorname{sgn} f\left(v_{i}\right)\right)_{i \in E}$ attained when varying over all linear functionals $f$ in $\left(\mathbb{R}^{d}\right)^{*}$; equivalently, they index the (relatively open) cones of all dimensions in the decomposition of $\mathbb{R}^{d}$ by the hyperplanes of $\mathcal{A}$. Then a strong map $N \rightarrow M$ abstracts the situation where $M$ comes from the image vectors $\left\{\varphi\left(v_{e}\right)\right\}_{e \in E}$ under some linear map $\mathbb{R}^{d} \stackrel{\varphi}{\rightarrow} \mathbb{R}^{d^{\prime}}$.

Richter-Gebert constructs in [24, §3] a certain rank 4 non-realizable, nonEuclidean oriented matroid that he calls $R(12)$, having 12 pseudohyperplanes in general position. He shows [24, Corollary 3.5] that there is a strong map from $R(12) \rightarrow M$, where $M$ is a uniform rank 2 oriented matroid, such that the topes (maximal covectors) of $M$ thought of as a subset of the topes of $R(12)$ cannot be contained in the topes of any pseudohyperplane that extends $R(12)$ by a single element (disproving a conjecture of M. Las Vergnas; see [24, Corollary 3.5]). 
Now pick $c_{0}$ to be any tope of $M$, which is necessarily also a tope of $R(12)$, and pick $r,-r$ to be the two unique minimal galleries from $c_{0}$ to $-c_{0}$ passing through topes of $M$, that is, $r,-r$ pass through the 12 hyperplanes of $M$ or $R(12)$ in exactly reversed orders. Hence if there existed a sequence of $\left(\begin{array}{c}12 \\ 2\end{array}\right)=\left|L_{2}(R(12))\right|$ elementary deformations connecting $r$ to $-r$, this would lead to a simple allowable sequence of permutations of length 12 in the sense of Goodman and Pollack; see 12, Chapter 6]. Such an allowable sequence would then give rise to the topes of a uniform rank 3 oriented matroid, containing the topes of $M$, and coming from a pseudohyperlane that extends $R(12)$ by a single element, contradicting Richter-Gebert's result.

Remark 2.8 (On the relation to monotone path zonotopes). We explain here how Billera and Sturmfels' theory of fiber polytopes [8] offers an enlightening perspective on the graph $G_{2}$, implying good behavior for certain of its subgraphs. The reader is referred to Ziegler [33, Lectures 7 and 9] for definitions and terminology omitted in this discussion.

Consider the (central, essential) arrangement $\mathcal{A}$ in $\mathbb{R}^{d}$ as the normal fan for the zonotope $Z(\mathcal{A})$ which is generated by functionals $\alpha_{H} \in\left(\mathbb{R}^{d}\right)^{*}$ that cut out the hyperplanes $H$, that is, it is the Minkowski sum $Z(\mathcal{A})=\sum_{H \in \mathcal{A}}\left[-\alpha_{H}, \alpha_{H}\right]$ of the line segments $\left[-\alpha_{H}, \alpha_{H}\right]$. Then the graph $G_{1}$ defined in Definition 2.1 is exactly the 1-skeleton of this zonotope $Z(\mathcal{A})$.

Now assume that the functionals $\alpha_{H}$ have been chosen to be positive on points in the chosen base chamber $c_{0}$ of $\mathcal{A}$. Then any point $f$ in (the interior of) $-c_{0}$ gives a linear functional that achieves its minimum, maximum values on $Z(\mathcal{A})$ at the vertices whose normal cones are the chambers $c_{0},-c_{0}$. Let $I=f(Z(\mathcal{A}))$ be the interval inside $\mathbb{R}$ which is the image of $Z(\mathcal{A})$ under this functional $f$. Then the fiber polytope/monotone path polytope

$$
Z_{2}:=\Sigma(Z(\mathcal{A}) \stackrel{f}{\rightarrow} I)=\Sigma_{f}(Z(\mathcal{A}))
$$

discussed by Billera and Sturmfels in [8, Theorem 5.3] is a $(d-1)$-dimensional polytope with several interesting properties.

The 1-skeleton of $Z_{2}$ turns out to be a certain subgraph of the graph $G_{2}$ from Definition 2.2. Specifically, minimal galleries $c_{0}$ to $-c_{0}$ correspond to $f$-monotone paths $\gamma$ in the 1 -skeleton of $Z(\mathcal{A})$. The vertices of $Z_{2}$ correspond to the subset of $f$-monotone paths $\gamma$ that are coherent, in the sense that there exists some linear functional $g$ whose maximum over each fiber $f^{-1}(x) \cap Z(\mathcal{A})$ for $x \in I$ is achieved uniquely at the point $f^{-1}(x) \cap \gamma$; see [8, Theorem 2.1].

Furthermore, 8, Theorem 2.4, Theorem 4.1] imply that $Z_{2}$ is a zonotope, generated by the vectors $\left\{v_{H, H^{\prime}}\right\}_{H \neq H^{\prime} \in \mathcal{A}}$ where $v_{H, H^{\prime}}:=f\left(\alpha_{H}\right) \alpha_{H^{\prime}}-f\left(\alpha_{H^{\prime}}\right) \alpha_{H}$. One can check that, for generic choices of $f$ within the interior of $-c_{0}$, any two such generating vectors $v_{H_{1}, H_{2}}$ and $v_{H_{3}, H_{4}}$ for $Z_{2}$ are scalar multiples of each other exactly when the codimension-two intersection subspaces $H_{1} \cap H_{2}$ and $H_{3} \cap H_{4}$ are the same subspace $X$ in $L_{2}$.

Hence $Z_{2}$ will then be a zonotope having exactly $\left|L_{2}\right|$ distinct parallelism classes among its generating vectors, and the 1-skeleton of $Z_{2}$ will be a geometrically distinguished subgraph of $G_{2}$ having the expected diameter $\left|L_{2}\right|$.

\section{Set-VAlued Metrics ON GRAPHS}

We introduce some easy observations that apply to the question of diameter for the graphs $G_{1}, G_{2}$ defined in the previous section. 
Definition 3.1. Let $G=(V, E)$ be a simple graph on vertex set $V$, meaning that $E$ is a set of unordered pairs $\{x, y\}$ with $x \neq y \in V$.

The graph-theoretic distance $d_{G}(x, y)$ is the minimum length $d$ of a path

$$
x=v_{0}, v_{1}, \ldots, v_{d-1}, v_{d}=y
$$

with $\left\{v_{i}, v_{i+1}\right\} \in E$ for each $i$. Call such a shortest path a geodesic.

The diameter of $G$ is the maximum value of $d_{G}(x, y)$ over all $x, y \in V$.

Note that $d_{G}(-,-)$ satisfies the usual properties of a metric on $V$, that is,

- $d_{G}(x, x)=0$,

- $d_{G}(x, y)=d_{G}(y, x)$, and

- $d_{G}(x, z) \leq d_{G}(x, y)+d_{G}(y, z)$.

We also make the trivial observation that if $\alpha: V \rightarrow V$ is a graph automorphism, meaning a bijection such that for every edge $\{x, y\}$ in $E$, the image $\{\alpha(x), \alpha(y)\}$ is also in $E$, then $\alpha$ takes geodesics to geodesics and preserves distances:

$$
d_{G}(\alpha(x), \alpha(y))=d_{G}(x, y) .
$$

Definition 3.2. For a connected simple graph $G=(V, E)$ and a set $\Omega$, say that a function

$$
\begin{aligned}
\Omega(-,-): V \times V & \longrightarrow 2^{\Omega} \\
(x, y) & \longmapsto \Omega(x, y)
\end{aligned}
$$

is a set-valued metric on $G$ if

(a) $\Omega(x, y)=\Omega(y, x)$,

(b) whenever $\{x, y\}$ is an edge in $E$, one has $|\Omega(x, y)|=1$, and

(c) $\Omega(x, z)=\Omega(x, y) \triangle \Omega(y, z)$,

where here

$$
A \triangle B:=(A \backslash B) \sqcup(B \backslash A)
$$

denotes the symmetric difference of sets. In particular, the first and third conditions imply that $\Omega(x, x)=\varnothing$ for any $x$ in $V$.

Here is an equivalent rephrasing of a set-valued metric $\Omega(-,-)$ on $G$ : it is a labelling $\Omega(x, y)$ of each edge $e=\{x, y\}$ in $E$ with an element of $\Omega$ in such a way that when one traverses any closed path of edges in the graph, each label appears an even number of times. For any pair of vertices $x, y$ in $V$, not necessarily connected by an edge, one defines $\Omega(x, y)$ to be the set of labels that appear an odd number of times on any path from $x$ to $y$.

Example 3.3. Given a real hyperplane arrangement $\mathcal{A}$, and the graphs $G_{i}$ for $i=1,2$ defined in Definitions 2.1 and 2.2, one can easily check that the function $L_{i}(-,-)$ for $i=1,2$ gives a set-valued metric.

We begin with two observations about set-valued metrics.

Proposition 3.4. A connected simple graph $G=(V, E)$ supports at least one setvalued metric if and only if $G$ bipartite.

Proof. Given a set-valued metric $\Omega(-,-)$ on $G$, choosing any vertex $x_{0}$ in $V$, one has that $G$ is bipartite with vertex bipartition $V=V_{0} \sqcup V_{1}$ in which

$$
V_{i}:=\left\{y \in V:\left|\Omega\left(x_{0}, y\right)\right| \equiv i \bmod 2\right\} .
$$


Conversely, for $G$ bipartite with vertex bipartition $V=V_{0} \sqcup V_{1}$, one can define a trivial set-valued metric $\Omega(-,-): V \times V \longrightarrow 2^{\{e\}}$, where $\{e\}$ is a singleton, via

$$
\Omega(x, y):= \begin{cases}\varnothing & \text { if } x, y \in V_{0} \text { or } x, y \in V_{1}, \\ \{e\} & \text { otherwise. }\end{cases}
$$

Proposition 3.5. A simple graph $G=(V, E)$ with a set-valued metric $\Omega(-,-)$, has $d_{G}(x, y) \geq|\Omega(x, y)|$ for all $x, y \in V$.

Proof. A path of length $d$ in $G$ from $x$ to $y$ as in (1) leads to a path

$$
\varnothing=\Omega\left(x, v_{0}\right), \Omega\left(x, v_{1}\right), \ldots, \Omega\left(x, v_{d-1}\right), \Omega\left(x, v_{d}\right)=\Omega(x, y),
$$

where each pair of sets $\Omega\left(x, v_{i}\right), \Omega\left(x, v_{i+1}\right)$ differs in one element, namely the unique element of $\Omega\left(v_{i}, v_{i+1}\right)$. Thus $d \geq|\Omega(x, y)|$.

Definition 3.6. For a set-valued metric $\Omega(-,-)$ on a simple graph $G=(V, E)$, say an involution $x \mapsto-x$ on the vertex set $V$ is $\left(\mathbb{Z}_{2}-\right)$ equivariant if

$$
\Omega(x,-y)=\Omega \backslash \Omega(x, y)
$$

for all $x, y$ in $V$. This is equivalent, by property (b) in Definition 3.2 of set-valued metrics, to requiring only the special case of (2) where $y=x$, that is, equivariance requires only $\Omega(x,-x)=\Omega$ for all $x$ in $V$.

Example 3.7. Continuing Example 3.3, the graphs $G_{i}$ for $i=1,2$ endowed with the set-valued metrics $L_{i}(-,-)$ also have equivariant involutions derived from the linear map $x \mapsto-x$ on $\mathbb{R}^{d}$. For $G_{1}$, the involution sends the chamber $c \in \mathcal{C}$ to the antipodal chamber $-c$. For $G_{2}$, the involution sends the minimal gallery $r$

$$
c_{0}, c_{1}, \ldots, c_{d-1}, c_{d}:=-c_{0}
$$

to the minimal gallery $-r$ which visits the antipodes of the same chambers in the reverse order:

$$
c_{0}=-c_{d},-c_{d-1}, \ldots,-c_{1},-c_{0} .
$$

Equivalently, $r$ and $-r$ cross the hyperplanes of $\mathcal{A}$ in exactly the opposite linear orders. For example, in the reflection arrangement of type $A_{n-1}$, one can check that this involution on galleries sends a reduced word $r=\left(s_{i_{1}}, s_{i_{2}}, \ldots, s_{i_{\ell-1}}, s_{i_{\ell}}\right)$ for $w_{0}$ to the reduced word $-r=\left(s_{n-i_{\ell}}, s_{n-i_{\ell-1}}, \ldots, s_{n-i_{2}}, s_{n-i_{1}}\right)$.

Proposition 3.8. A simple graph $G=(V, E)$ with a set-valued metric $\Omega(-,-)$ and an equivariant involution always has diameter at least $|\Omega|$.

Proof. By Proposition 3.5

$$
d_{G}(x,-x) \geq|\Omega(x,-x)|=|\Omega \backslash \Omega(x, x)|=|\Omega| .
$$

Definition 3.9. For a set-valued metric $\Omega(-,-)$ on a simple graph $G=(V, E)$, say that a vertex $x_{0}$ in $V$ is $\Omega$-accessible if $d_{G}\left(x_{0}, y\right)=\left|\Omega\left(x_{0}, y\right)\right|$ for every $y \in V$.

Example 3.10. Continuing Examples 3.3 and 3.7, it was observed by Edelman (see [16, Proposition 1.1]) that for every real hyperplane arrangement $\mathcal{A}$, every vertex in the graph $G_{1}$ is $L_{1}$-accessible: given any two chambers $c, c^{\prime}$, a straightline path between generic points in $c, c^{\prime}$ gives a path of length $\left|L_{1}\left(c, c^{\prime}\right)\right|$ between their corresponding vertices of $G_{1}$. 
Example 3.11. Of the 16 reduced words for $w_{0}$ in $W=\mathfrak{S}_{4}$ shown in Figure 1.1, there are exactly four which do not give $L_{2}$-accessible vertices for the graph $G_{2}$ shown, namely the four words

$$
\{213213, \quad 231231, \quad 132132, \quad 312312\} \text {. }
$$

We check that none of the four words $r_{0}$ in this set (3) is $L_{2}$-accessible. Scrutiny of Figures 1.1 and 2.2 shows that there are exactly two words at the maximum distance $7\left(=\left|L_{2}\right|\right)$ from such an $r_{0}$, namely its antipodal word $-r_{0}$, and a second word $r \neq-r_{0}$ having $d_{G_{2}}\left(r_{0}, r\right)=7>5=\left|L_{2}\left(r_{0}, r\right)\right|$. In particular, any such pair $\left\{r_{0}, r\right\}$ provides an example that answers negatively a question of Autord and Dehornoy [4, Question 1.9].

On the other hand, one can check using Figures 1.1 and 2.2, via brute force (mitigated by some symmetry), that all 12 of the other words $r_{0}$ are $L_{2}$-accessible.

Our goal in Section 4 will be to show that for supersolvable arrangements $\mathcal{A}$, when one chooses the base chamber $c_{0} \in \mathcal{C}$ incident to a chain of modular flats, there is a choice of base gallery $r_{0} \in \mathcal{R}$ which is $L_{2}$-accessible. Therefore, in this case, the diameter for $G_{2}$ will be determined by the next proposition.

Proposition 3.12. Assume one has a simple graph $G=(V, E)$ with a set-valued metric $\Omega(-,-)$, and an involution $v \mapsto-v$ on $V$ which is both equivariant and $a$ graph automorphism of $G$.

If $V$ contains an $\Omega$-accessible vertex $x_{0}$, then the diameter of $G$ is exactly $|\Omega|$.

Proof. By Proposition 3.8, it suffices to show that $d_{G}(x, y) \leq|\Omega|$ for all $x, y$. This follows from these equalities and inequalities, justified below:

$$
\begin{aligned}
2 d_{G}(x, y) & =d_{G}(x, y)+d_{G}(x, y) \\
& \stackrel{(1)}{\leq} d_{G}\left(x, x_{0}\right)+d_{G}\left(x_{0}, y\right)+d_{G}\left(x,-x_{0}\right)+d_{G}\left(-x_{0}, y\right) \\
& \stackrel{(2)}{=} d_{G}\left(x, x_{0}\right)+d_{G}\left(x_{0}, y\right)+d_{G}\left(-x, x_{0}\right)+d_{G}\left(x_{0},-y\right) \\
& =\left(d_{G}\left(x, x_{0}\right)+d_{G}\left(-x, x_{0}\right)\right)+\left(d_{G}\left(x_{0}, y\right)+d_{G}\left(x_{0},-y\right)\right) \\
& \stackrel{(3)}{=}\left(\left|\Omega\left(x, x_{0}\right)\right|+\left|\Omega\left(-x, x_{0}\right)\right|\right)+\left(\left|\Omega\left(x_{0}, y\right)\right|+\left|\Omega\left(x_{0},-y\right)\right|\right) \\
& \stackrel{(4)}{=}|\Omega|+|\Omega|=2|\Omega| .
\end{aligned}
$$

Inequality (1) twice uses the triangle inequality for the metric $d_{G}(-,-)$. Equality (2) twice uses the assumption that $v \mapsto-v$ is a a graph automorphism. Equality (3) four times uses the $\Omega$-accessibility of $x_{0}$. Equality (4) twice uses the assumption of equivariance.

Applying Propositions 3.8 and 3.12 to the graphs $G_{1}, G_{2}$ immediately gives the following.

Corollary 3.13. For any real hyperplane arrangement $\mathcal{A}$, the graph $G_{1}$ has diameter exactly $\left|L_{1}\right|$. For any choice of a base chamber $c_{0}$ of $\mathcal{A}$, the graph $G_{2}$ has diameter at least $\left|L_{2}\right|$.

\section{Supersolvable arrangements}

We wish to first review the definition and some properties of supersolvable arrangements [27, [20, §2.3], [11, §4], and then apply this to prove Theorem [1.1. 
Definition 4.1. Given a finite geometric lattice $L$ with rank function $\rho$, say that $x$ in $L$ a modular element of $L$ if $\rho(x \vee y)+\rho(x \wedge y)=\rho(x)+\rho(y)$ for all $y$ in $L$. The lattice $L$ is called supersolvable if it contains an $M$-chain, that is, a maximal chain of modular elements.

A hyperplane arrangement $\mathcal{A}$ is called supersolvable when its intersection lattice $L$ is supersolvable. An inductive rephrasing, due to Björner, Edelman and Ziegler [11, Thm. 4.3], will be more useful for our purposes. Given a real (central, essential) hyperplane arrangement $\mathcal{A}$ in $\mathbb{R}^{d}$, an element $\ell$ of $L_{d-1}$ is called a coatom. Thus coatoms $\ell$ are lines obtained by intersecting the hyperplanes.

Proposition 4.2 ([11, Thm. 4.3]). Let $\mathcal{A}$ be a hyperplane arrangement.

(i) A coatom $\ell$ of $L$ is a modular coatom if and only if for every pair $H, H^{\prime}$ of distinct hyperplanes of $\mathcal{A}$ not containing $\ell$ there exists a hyperplane $H^{\prime \prime}$ of $\mathcal{A}$ containing both $\ell$ and $H \cap H^{\prime}$ (that is to say, the hyperplane $H^{\prime \prime}=\ell+H \cap H^{\prime}$ is in $\mathcal{A})$.

(ii) A hyperplane arrangement $\mathcal{A}$ is supersolvable if and only if it satisfies the following inductive definition: either $\mathcal{A}$ has rank $d=1$ or its intersection lattice $L$ contains a modular coatom $\ell$ for which the localized arrangement $\mathcal{A}_{\ell}$ of rank $d-1$ is supersolvable.

Example 4.3. When $d=2$ the arrangement $\mathcal{A}$ is always supersolvable, as any of its hyperplanes (=lines) is a modular coatom.

Example 4.4. Recall from Example 2.4 that the reflection arrangement $\mathcal{A}$ of type $A_{n-1}$, corresponding to $W=\mathfrak{S}_{n}$, lives in $\mathbb{R}^{n} /\left\{x_{1}=x_{2}=\cdots=x_{n}\right\}$, and has hyperplanes $H_{i j}:=\left\{x_{i}=x_{j}\right\}$. The line $\ell$ defined by $\left\{x_{1}=x_{2}=\cdots=x_{n-1}\right\}$ is a modular coatom for $A_{n-1}$ : any two typical hyperplanes $H_{i n}, H_{j n}$ for $i, j<n$ that do not contain $\ell$ will have $\ell+\left(H_{i n} \cap H_{j n}\right)=H_{i j}$, which is another hyperplane in the arrangement $A_{n-1}$. The localization $\mathcal{A}_{\ell}$ is isomorphic to the reflection arrangement of type $A_{n-2}$, and hence one can iterate this construction to show that the arrangement of type $A_{n-1}$ is supersolvable.

Example 4.5. The reflection arrangement $\mathcal{A}$ of type $B_{n}$ lives in $\mathbb{R}^{n}$, and consists of all hyperplanes of the form

$$
\begin{aligned}
& H_{i j}^{+}:=\left\{x_{i}=+x_{j}\right\}, \\
& H_{i j}^{-}:=\left\{x_{i}=-x_{j}\right\}, \\
& H_{i i}^{-}:=\left\{x_{i}=0\right\} .
\end{aligned}
$$

Let us check that the line $\ell$ defined by $\left\{x_{1}=x_{2}=\cdots=x_{n-1}=0\right\}$ is a modular coatom for $B_{n}$. Pairs of hyperplanes not containing $\ell$ either come from choosing

- two indices $i, j \leq n-1$ and two signs $\alpha, \beta= \pm 1$, giving hyperplanes $H_{i n}^{\alpha}, H_{j n}^{\beta}$ which satisfy $\ell+\left(H_{i n}^{\alpha} \cap H_{j n}^{\beta}\right)=H_{i j}^{\alpha \cdot \beta}$, a hyperplane of $B_{n}$; or

- an index $i \leq n-1$ and a sign $\alpha$, giving hyperplanes $H_{n n}^{-}, H_{i n}^{\alpha}$, which satisfy $\ell+\left(H_{n n}^{-} \cap H_{i n}^{\alpha}\right)=H_{i i}^{-}$, a hyperplane of $B_{n}$.

The localization $\mathcal{A}_{\ell}$ is isomorphic to the reflection arrangement of type $B_{n-1}$, and hence one can iterate this construction to show that the arrangement of type $B_{n}$ is supersolvable. 
Given an intersection subspace $X$, define the map $\pi_{X}$ sending chambers of $\mathcal{A}$ to their corresponding chamber in the localization $\mathcal{A}_{X}$ :

$$
\begin{aligned}
\pi_{X}: \mathcal{C}=: \mathcal{C}(\mathcal{A}) & \longrightarrow \mathcal{C}\left(\mathcal{A}_{X}\right) \\
c & \longmapsto c / X .
\end{aligned}
$$

Say that a chamber $c$ is incident to a subspace $X$ if the closure of $c$ intersects $X$ in a subcone of the same dimension as $X$.

Proposition 4.6. Assume that $\ell$ is a modular coatom for $\mathcal{A}$, and $c \in \mathcal{C}$ is a chamber incident to $\ell$.

(i) (Compare with the discussion before Theorem 4.4 of [1]) There is a linear order on the fiber

$$
\pi_{\ell}^{-1}\left(\pi_{\ell}(c)\right)=\left\{c_{1}(=c), c_{2}, c_{3}, \ldots, c_{t}\right\}
$$

such that the sets $L_{1}\left(c, c_{i}\right)$ for $i=1,2, \ldots, t$ are nested:

$$
\varnothing=L_{1}\left(c, c_{1}\right) \subset L_{1}\left(c, c_{2}\right) \subset \cdots \subset L_{1}\left(c, c_{t}\right)=\mathcal{A} \backslash \mathcal{A}_{\ell} .
$$

This induces a linear order $H_{1}, H_{2}, \ldots$ on $\mathcal{A} \backslash \mathcal{A}_{\ell}$ such that $H_{i}$ is the unique hyperplane in $L_{1}\left(c, c_{i}\right) \backslash L_{1}\left(c, c_{i-1}\right)$.

(ii) Using the linear order $H_{1}, H_{2}, \ldots$ on $\mathcal{A} \backslash \mathcal{A}_{\ell}$ from part (i), if $i<j<k$ and if the chamber $c$ incident to $\ell$ is also incident to $\ell+H_{i} \cap H_{k}$, then

$$
H_{i} \cap H_{j}=H_{i} \cap H_{k}=H_{j} \cap H_{k} .
$$

Proof of assertion (i). Assume for the sake of contradiction that there exist two chambers $c_{i}, c_{j}$ with $\pi_{\ell}\left(c_{i}\right)=\pi_{\ell}\left(c_{j}\right)=\pi_{\ell}(c)$ and two hyperplanes $H_{i}, H_{j}$ not containing $\ell$ for which

$$
\begin{aligned}
& H_{i} \in L_{1}\left(c, c_{i}\right) \backslash L_{1}\left(c, c_{j}\right), \\
& H_{j} \in L_{1}\left(c, c_{j}\right) \backslash L_{1}\left(c, c_{i}\right) .
\end{aligned}
$$

By the modularity of the coatom $\ell$, the hyperplane $H:=\ell+H_{i} \cap H_{j}$ is in $\mathcal{A}$. Consider the codimension-two subspace

$$
X:=H_{i} \cap H_{j}=H \cap H_{i}=H \cap H_{j}
$$

and the local picture for the lines and chambers

$$
H / X, H_{i} / X, H_{j} / X, c / X, c_{i} / X, c_{j} / X
$$

within the localized rank two arrangement $\mathcal{A}_{X}$. Then (5) together with the assumption that $H$ contains the line $\ell$ incident to $c$ forces this local picture to be as in Figure 4.1(i). In particular, it forces $H$ to separate $c_{i}, c_{j}$, and since $\ell \subset H$, this contradicts $\pi_{\ell}\left(c_{i}\right)=\pi_{\ell}\left(c_{j}\right)$.

Proof of assertion (ii). Assume for the sake of contradiction that $i<j<k$ and $c$ is incident to $\ell+H_{i} \cap H_{k}$, but (4) fails, so that the intersection $X:=H_{i} \cap H_{j} \cap H_{k}$ is of codimension-three, not two. Note that $\ell$ is contained in none of $H_{i}, H_{j}, H_{k}$. Therefore since $\ell$ is a modular coatom, each of the following three hyperplanes containing both $\ell$ and $X$ must also be a hyperplane in $\mathcal{A}$ :

$$
\begin{aligned}
H_{i j} & :=\ell+H_{i} \cap H_{j}, \\
H_{i k} & :=\ell+H_{i} \cap H_{k}, \\
H_{j k} & :=\ell+H_{j} \cap H_{k} .
\end{aligned}
$$


(i)

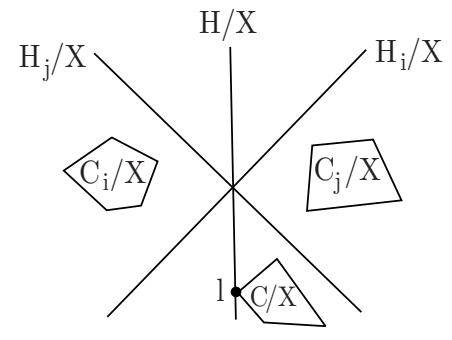

(ii)

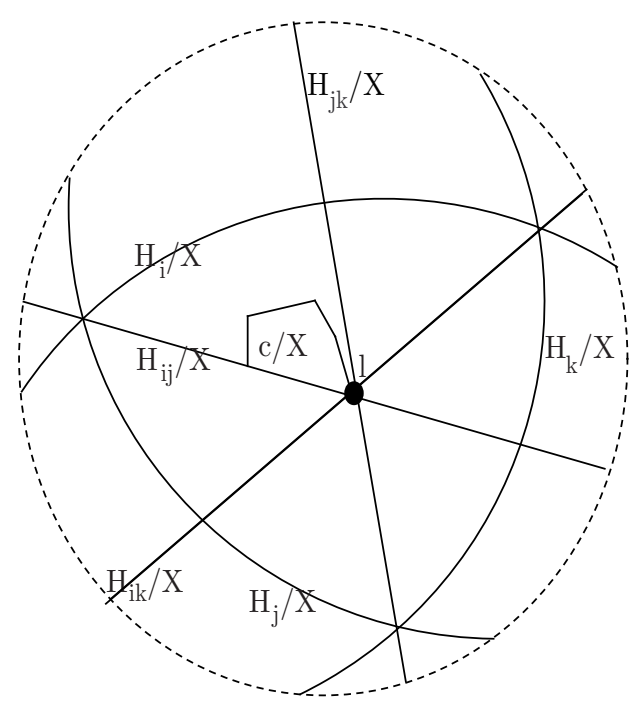

Figure 4.1. (i) Local picture illustrating why the fibers $\pi_{\ell}^{-1}\left(\pi_{\ell}(c)\right)$ are linearly ordered by inclusion of $L(c,-)$.

(ii) Local picture illustrating why incidence of $c$ to $\ell+H_{i} \cap H_{k}$ and $i<j<k$ forces the equality (4).

Now consider the local picture for

$$
H_{i} / X, H_{j} / X, H_{k} / X, H_{i j} / X, H_{i k} / X, H_{j k} / X, \ell / X
$$

within $\mathcal{A}_{X}$, which after an invertible linear transformation of $\mathbb{R}^{d} / X$, can be made to look as in Figure 4.1(ii).

Recalling that $c$ is incident to the line $\ell$, the condition $i<j<k$, forces $c / X$ to be in the chamber shown, so that as one starts in $c$ and moves away from $\ell$ staying within the same chamber of $\mathcal{A}_{\ell}$, one crosses the hyperplanes $H_{i}, H_{j}, H_{k}$ in this order; if $c / X$ lies in any of the other five chambers incident to $\ell / X$ in this figure, one will cross $H_{i}, H_{j}, H_{k}$ in a different order.

However, Figure 4.1(ii) also shows that this location for $c / X$ contradicts the incidence of $c$ to $H_{i k}=\ell+H_{i} \cap H_{k}$.

Definition 4.7. A (maximal) flag $F=\left\{X_{i}\right\}_{i=0}^{d}$ is a chain of intersection subspaces in $L$

$$
\{0\}=X_{0} \subset X_{1} \subset X_{2} \subset \cdots \subset X_{d-1} \subset X_{d}=\mathbb{R}^{d}
$$


in which $X_{i}$ is of dimension $i$. Say that a chamber $c$ is incident to the flag $F$ if $c$ is incident to each of the $X_{i}$. If $c_{0}$ is a chamber incident to a flag $F$, say that a minimal gallery $r$ from $c_{0}$ to $-c_{0}$ is incident to the flag $F$ if it first crosses the unique hyperplane in $\mathcal{A}_{X_{d-1}}$ (namely $X_{d-1}$ itself), then crosses the hyperplanes in $\mathcal{A}_{X_{d-2}} \backslash \mathcal{A}_{X_{d-1}}$, etc., always crossing the hyperplanes in $\mathcal{A}_{X_{j}}$ before those in $\mathcal{A}_{X_{i}} \backslash \mathcal{A}_{X_{j}}$ whenever $i<j$.

According to the definition of supersolvability (Definition 4.1) and its rephrasing in Proposition 4.2 $\mathcal{A}$ is supersolvable if and only if it has an $M$-chain or modular flag, in which $X_{1}$ is a modular coatom for $\mathcal{A}$, while $X_{2} / X_{1}$ is a modular coatom in the localized arrangement $\mathcal{A}_{X_{1}}$, and generally $X_{i} / X_{i-1}$ is a modular coatom in the localized arrangement $\mathcal{A}_{X_{i-1}}$.

Proposition 4.8. Let $\ell$ be a coatom of the intersection lattice $L$ for an arrangement A. The map

$$
\begin{gathered}
\mathcal{C}(\mathcal{A}) \stackrel{\pi_{\ell}}{\longrightarrow} \mathcal{C}\left(\mathcal{A}_{\ell}\right) \\
c \longmapsto c / \ell
\end{gathered}
$$

becomes two-to-one when restricted to the subset of chambers of $\mathcal{A}$ incident to $\ell$.

Consequently 3 when $\mathcal{A}$ is a real central essential arrangement in $\mathbb{R}^{d}$, there are exactly $2^{d}$ chambers incident to each maximal flag $F$.

Proof. The two chambers of a fiber $\pi_{\ell}^{-1}\left(\pi_{\ell}(c)\right)$ that contain $\ell$ are the two chambers $c^{+}, c^{-}$in the fiber whose closures contain $\ell^{+}, \ell^{-}$, the two rays (half-lines) comprising the line $\ell$.

Theorem 4.9. Let $\mathcal{A}$ be a real (central, essential) hyperplane arrangement in $\mathbb{R}^{d}$ which is supersolvable, let $F:=\left\{X_{i}\right\}_{i=0}^{d}$ be a modular flag for $\mathcal{A}$, and let $c_{0}$ be any chamber incident to $F$.

(i) There is a unique minimal gallery $r_{0}$ from $c_{0}$ to $-c_{0}$ incident to $F$.

(ii) This minimal gallery $r_{0}$ is an $L_{2}$-accessible vertex for the graph $G_{2}$ on the galleries $\mathcal{R}$ from $c_{0}$ to $-c_{0}$.

Proof of assertion (i). Proceed by induction on $d$, with the base case $d=1$ being trivial. In the inductive step, let $\ell^{+}$be the half-line of $\ell$ contained in the closure of $c_{0}$. Then the unique minimal gallery $r_{0}$ from $c_{0}$ to $-c_{0}$ incident to $F$ is constructed as follows, in order to have it cross all the hyperplanes in $\mathcal{A}_{\ell}$ first:

(a) Apply induction to the $(d-1)$-dimensional supersolvable arrrangement $\mathcal{A}_{\ell}$, to find the unique gallery from $c_{0} / \ell$ to $-c_{0} / \ell$ incident to $F / \ell$.

(b) Begin the gallery $r_{0}$ by lifting each chamber $c / \ell$ of $\mathcal{A}_{\ell}$ in this gallery from (a) to the unique chamber $c$ in $\mathcal{A}$ whose closure contains $\ell^{+}$.

(c) After going through the chambers in this lifted gallery from (b), ending in a gallery called $c$ incident to $\ell$, one now has no choice about how to complete the rest of $r_{0}$ : one must cross the hyperplanes $\mathcal{A} \backslash \mathcal{A}_{\ell}$ in the linear order given by Proposition 4.6(i).

For example, for the reflection arrangement of type $A_{3}$, the base word/gallery $r_{0}=121321$, indexing the vertex at the bottom left in Figure 1.1 is the unique gallery $r_{0}$ incident to the modular flag described in Example 4.4; this gallery is discussed further below in Example4.10. Figure 4.2 shows the reflection arrangement of type $B_{3}$, with the unique gallery $r_{0}$ incident to a certain modular flag labeled.

\footnotetext{
${ }^{3}$ Compare this with the discussion before Theorem 4.4 of [1].
} 


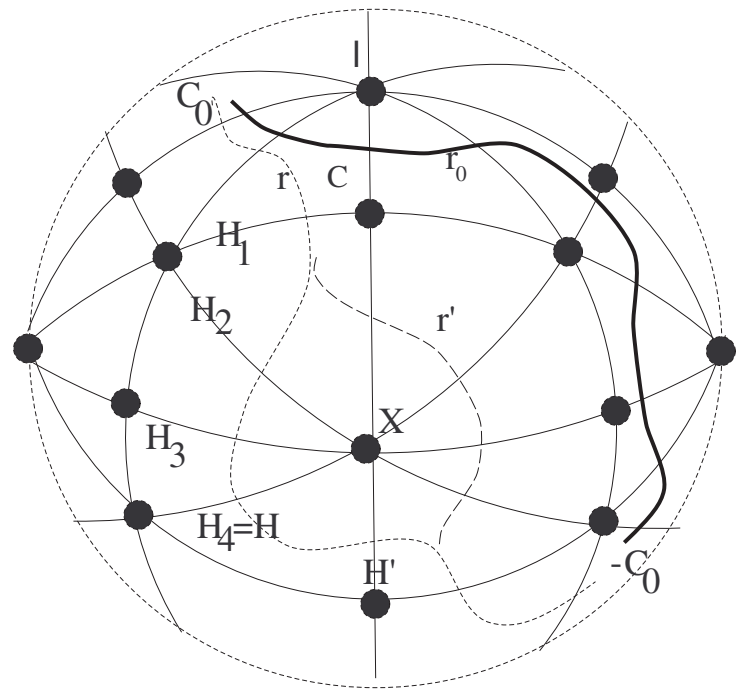

FIgURE 4.2. The reflection arrangement of type $B_{3}$, drawn as great circles on a unit sphere. The chamber $c_{0}$ and its antipode $-c_{0}$ are labeled, as well as the three galleries $r_{0}, r, r^{\prime}$ from $c_{0}$ to $-c_{0}$ which appear in the proof of Theorem 4.9 .

Proof of assertion (ii). Proceed by induction on $d$, with the base case $d=2$ being trivial. In the inductive step, it suffices to show that for any gallery $r \neq r_{0}$ from $-c_{0}$ to $c_{0}$, there exists another gallery $r^{\prime}$ having $L_{2}\left(r_{0}, r^{\prime}\right) \subset L_{2}\left(r_{0}, r\right)$ and $\left|L_{2}\left(r, r^{\prime}\right)\right|=1$. There are two cases.

Case 1 . The gallery $r$ crosses all the hyperplanes in $\mathcal{A}_{\ell}$ before crossing any hyperplanes of $\mathcal{A} \backslash \mathcal{A}_{\ell}$.

Then just as with $r_{0}$, the gallery $r$ must cross the hyperplanes in $\mathcal{A} \backslash \mathcal{A}_{\ell}$ in the linear order given by Proposition 4.6. Hence the galleries $r$ and $r_{0}$ can differ only in their initial segments $\hat{r}, \hat{r_{0}}$ where they cross all the hyperplanes of $\mathcal{A}_{\ell}$. Applying induction on dimension to the quotient galleries $\hat{r} / \ell, \hat{r_{0}} / \ell$, there exists a gallery $\hat{r^{\prime}} / \ell$ from $c_{0} / \ell$ to $-c_{0} / \ell$ in $\mathcal{A}_{\ell}$ having $L_{2}\left(\hat{r_{0}} / \ell, \hat{r^{\prime}} / \ell\right) \subset L_{2}\left(\hat{r_{0}} / \ell, \hat{r} / \ell\right)$ and $\left|L_{2}\left(\hat{r} / \ell, \hat{r}^{\prime} / \ell\right)\right|=1$. The desired gallery $r^{\prime}$ from $c_{0}$ to $-c_{0}$ is then obtained by first lifting $\hat{r}^{\prime} / \ell$ as in Proposition 4.8, and then completing it by crossing the hyperplanes in $\mathcal{A} \backslash \mathcal{A}_{\ell}$ in the linear order given by Proposition 4.6.

Case 2. The gallery $r$ crosses some hyperplane of $\mathcal{A} \backslash \mathcal{A}_{\ell}$ before it has finished crossing all the hyperplanes in $\mathcal{A}_{\ell}$.

Then there must exist at least one ordered pair of hyperplanes $\left(H, H^{\prime}\right)$ crossed consecutively by $r$ that has $H \in \mathcal{A} \backslash \mathcal{A}_{\ell}$ and $H^{\prime} \in \mathcal{A}_{\ell}$. Find the earliest occurrence 4 of such a pair $\left(H, H^{\prime}\right)$.

Thus $r$ begins by crossing some (possibly empty) sequence of hyperplanes in $\mathcal{A}_{\ell}$, reaching some chamber $c$ incident to $\ell$, and then immediately thereafter crosses a

\footnotetext{
${ }^{4}$ Compare the rest of this proof with the proof of [4, Lemma 1.2], which it generalizes.
} 
sequence of hyperplanes $H_{1}, H_{2}, \ldots, H_{t-1}, H_{t}=: H$ that are all in $\mathcal{A} \backslash \mathcal{A}_{\ell}$, before crossing $H^{\prime}$. See Figure 4.2 for an illustration of a typical gallery $r$, with the chamber $c$ and the hyperplanes $H_{1}, H_{2}, \ldots, H_{t-1}, H_{t}(=: H), H^{\prime}$ labeled as in this proof; in this example, $t=4$.

Let $X:=H \cap H^{\prime}$, a codimension-two subspace in $L_{2}$. Note that as $H^{\prime}=\ell+X$, this $H^{\prime}$ is the unique hyperplane in the rank 2 subarrangement $\mathcal{A}_{X}$ that also lies in $\mathcal{A}_{\ell}$. We wish to determine exactly when the other hyperplanes $\mathcal{A}_{X} \backslash\left\{H^{\prime}\right\}$ are crossed by the gallery $r$.

Note that since the quotient gallery $r / \ell$ would cross $H^{\prime} / \ell$ to leave $c / \ell$, this chamber $c / \ell$ must be incident to $H^{\prime} / \ell$. As $c$ is incident to $\ell$, this means that $c$ is incident to $H^{\prime}$.

Now since $r$ visits the chamber $c$ which is incident to $H^{\prime}$, but then crosses another hyperplane $H$ that contains $X$ before crossing $H^{\prime}$, it must be that $r$ crosses every other hyperplane of the rank 2 arrangement $\mathcal{A}_{X}$ before crossing $H^{\prime}$.

On the other hand, since $r$ only crossed hyperplanes in $\mathcal{A}_{\ell}$ before reaching $c$, it must be that

$$
\mathcal{A}_{X} \backslash\left\{H^{\prime}\right\} \subset\left\{H_{1}, H_{2}, \ldots, H_{t-1}, H_{t}(=H)\right\} .
$$

Also note that the hypotheses of Proposition 4.6(ii) are satisfied by $c, \ell$ and by any two hyperplanes $H_{i}, H_{k}$ lying in $\mathcal{A}_{X} \backslash\left\{H^{\prime}\right\}$, since $c$ is incident to

$$
H^{\prime}=\ell+X=\ell+H_{i} \cap H_{k} .
$$

This means that for any $j$ with $i<j<k$ one has $H_{j}$ also in $\mathcal{A}_{X} \backslash\left\{H^{\prime}\right\}$. Thus the hyperplanes of $\mathcal{A}_{X} \backslash\left\{H^{\prime}\right\}$ occur consecutively within the list $\left(H_{1}, \ldots, H_{t-1}, H_{t}\right)$. That is, we have shown that there is some index $s \leq t$ for which

$$
\left\{H_{s}, H_{s+1}, \ldots, H_{t-1}, H_{t}(=H), H^{\prime}\right\}=\mathcal{A}_{X} .
$$

Now let $r^{\prime}$ be the gallery obtained from $r$ by reversing this consecutive sequence of crossings $\left(H_{s}, H_{s+1}, \ldots, H_{t-1}, H_{t}(=H), H^{\prime}\right)$ of the hyperplanes in $\mathcal{A}_{X}$. By construction, $L_{2}\left(r, r^{\prime}\right)=\{X\}$.

We must also check that $L_{2}\left(r_{0}, r^{\prime}\right) \subset L_{2}\left(r_{0}, r\right)$, i.e. that $X$ is not in $L_{2}\left(r_{0}, r^{\prime}\right)$. This follows because $r_{0}$ is incident to $F$, so it must cross the hyperplane $H^{\prime} \in \mathcal{A}_{\ell}$ before it can cross the hyperplane $H \in \mathcal{A} \backslash \mathcal{A}_{\ell}$. Thus in regard to its order of crossing the hyperplanes of $\mathcal{A}_{X}$, the gallery $r_{0}$ agrees with $r^{\prime}$, not with $r$.

Theorem 1.1 is now immediate from Theorem 4.9(ii) and Proposition 3.12 .

Example 4.10. Continuing Example 4.4, for the arrangement $\mathcal{A}$ of type $A_{n-1}$, one can choose as modular flag $F:=\left\{X_{i}\right\}_{i=0}^{n-1}$, where

$$
X_{i}:=\left\{x_{1}=x_{2}=\cdots=x_{n-i}\right\}
$$

The chambers $c_{w} \in \mathcal{C}$ may be indexed by permutations $w$ in $\mathfrak{S}_{n}$, with defining inequalities $x_{w(1)}<\cdots<x_{w(n)}$. The chamber $c_{0}$ corresponding to the identity permutation is incident to the above modular flag $F$. The unique gallery $r_{0}$ from 
$c_{0}$ to $-c_{0}$ incident to $F$ crosses the hyperplanes in this order:

$$
\begin{aligned}
& H_{12}, \\
& H_{13}, H_{23}, \\
& H_{14}, H_{24}, H_{34}, \\
& H_{15}, H_{25}, H_{35}, H_{45}, \ldots .
\end{aligned}
$$

Using the Coxeter generators $S=\left\{s_{1}, \ldots, s_{n-1}\right\}$ for $W=\mathfrak{S}_{n}$, in which $s_{i}$ is the adjacent transposition $(i, i+1)$, this gallery $r_{0}$ corresponds to the following reduced decomposition for $w_{0}$ :

$$
\begin{aligned}
& \left(s_{1},\right. \\
& s_{2}, s_{1}, \\
& s_{3}, s_{2}, s_{1}, \\
& \left.s_{4}, s_{3}, s_{2}, s_{1}, \ldots\right) .
\end{aligned}
$$

Example 4.11. Continuing Example 4.5, for the arrangement $\mathcal{A}$ of type $B_{n}$, one can choose as modular flag $F:=\left\{X_{i}\right\}_{i=0}^{n}$, where

$$
X_{i}:=\left\{x_{1}=x_{2}=\cdots=x_{n-i}=0\right\} .
$$

The chambers $c_{w} \in \mathcal{C}$ may be indexed by signed permutations $w$ with defining inequalities $0<\epsilon_{1} x_{w(1)}<\cdots<\epsilon_{n} x_{w(n)}$ if $w$ sends the standard basis vector $e_{j}$ to $\epsilon_{j} e_{w(j)}$ with $\epsilon_{j} \in\{ \pm 1\}$. The chamber $c_{0}$ corresponding to the identity permutation is incident to the above modular flag $F$. The unique gallery $r_{0}$ from $c_{0}$ to $-c_{0}$ incident to $F$ crosses the hyperplanes in this order:

$$
\begin{aligned}
& H_{11}^{-}, \\
& H_{12}^{-}, H_{22}^{-}, H_{12}^{+}, \\
& H_{23}^{-}, H_{13}^{-}, H_{33}^{-}, H_{13}^{+}, H_{23}^{+}, \\
& H_{34}^{-}, H_{24}^{-}, H_{14}^{-}, H_{44}^{-}, H_{14}^{+}, H_{24}^{+}, H_{34}^{+}, \ldots .
\end{aligned}
$$

Choose Coxeter generators $S=\left\{s_{0}, s_{1}, \ldots, s_{n-1}\right\}$ for the hyperoctahedral group $W=B_{n}$ of signed permutations acting on the coordinates of $\mathbb{R}^{n}$, such that $s_{i}$ is the adjacent transposition $(i, i+1)$, as before, and $s_{0}$ is the sign change in the first coordinate $x_{1}$. Then this gallery $r_{0}$ corresponds to the following reduced decomposition for $w_{0}$ :

$$
\begin{aligned}
& \left(s_{0},\right. \\
& s_{1}, s_{0}, s_{1}, \\
& s_{2}, s_{1}, s_{0}, s_{1}, s_{2}, \\
& \left.s_{3}, s_{2}, s_{1}, s_{0}, s_{1}, s_{2}, s_{3}, \ldots\right) .
\end{aligned}
$$

Remark 4.12. Consider the following possible hypotheses on a real (central, essential) hyperplane arrangement $\mathcal{A}$ and one of its chambers $c_{0}$ :

(i) The chamber $c_{0}$ is a simplicial cone in the sense that its walls have linearly independent normal vectors.

(ii) The weak order on the chambers $\mathcal{C}$, considered by Edelman [16, in which $c \leq c^{\prime}$ when $L_{1}\left(c_{0}, c\right) \subseteq L_{1}\left(c_{0}, c^{\prime}\right)$, is a lattice. 
(iii) $\mathcal{A}$ is simplicial, meaning that every chamber is a simplicial cone.

(iv) $\mathcal{A}$ is a real reflection arrangement.

(v) The arrangement $\mathcal{A}$ is supersolvable, and the chamber $c_{0}$ is incident to one of its modular flags.

Incorporating well-known results for reflection arrangements with various results from Björner, Edelman and Ziegler [11, one has the following implications:

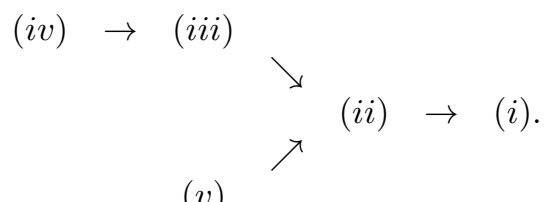

Bearing in mind that Theorem 1.1 assumes hypothesis (v), and the $d$-vertexconnectivity of the graph $G_{2}$ proven in [3. Theorem 1.1] assumes hypothesis (iii), it is reasonable to ask whether any of the extra hypotheses (i), (ii), (iii), (iv) imply that the lower bound of $\left|L_{2}\right|$ for the diameter of the graph $G_{2}$ is tight.

\section{ON THE GRAPHS $G(w)$}

In this section we show how the previous methods generalize to the graph $G(w)$ of reduced words for $w$ an element of a finite reflection group $W$, as discussed in the Introduction. Although these methods lead to some bounds on the distance functions and diameters of $G(w)$ in the groups of type $A, B$, we do not determine these diameters exactly.

As in Section 2, let $\mathcal{A}$ be a (central, essential) hyperplane arrangement in $\mathbb{R}^{d}$, with set of chambers $\mathcal{C}$. Given two chambers $c, c^{\prime} \in \mathcal{C}$, recall that $L_{1}\left(c, c^{\prime}\right)$ denotes the set of hyperplanes $H \in L_{1}=\mathcal{A}$ that separate $c$ from $c^{\prime}$ or, equivalently, for which the chambers $c / H, c^{\prime} / H$ in the localized rank 1 arrangement $\mathcal{A}_{H}$ are antipodal. Further define

$$
\begin{aligned}
L_{2}\left(c, c^{\prime}\right): & =\left\{X \in L_{2}: c / X, c^{\prime} / X \text { are antipodal chambers in } \mathcal{A}_{X}\right\} \\
& =\left\{X \in L_{2}: \mathcal{A}_{X} \subseteq L_{1}\left(c, c^{\prime}\right)\right\} .
\end{aligned}
$$

Denote by $\mathcal{R}\left(c, c^{\prime}\right)$ the set of all minimal galleries $r$ from $c$ to $c^{\prime}$.

Note that any minimal gallery $r$ in $\mathcal{R}\left(c, c^{\prime}\right)$ must cross each of the hyperplanes in $L_{1}\left(c, c^{\prime}\right)$ exactly once, and is completely determined by the linear order in which these hyperplanes are crossed. For each codimension-two subspace $X$ in $L_{2}$, there are two possibilities:

- If $X \notin L_{2}\left(c, c^{\prime}\right)$, then the hyperplanes of $\mathcal{A}_{X}$ must be crossed by $r$ in a unique linear order, namely the order in which one crosses the hyperplanes of $\mathcal{A}_{X} \cap L_{1}\left(c, c^{\prime}\right)$ when walking from $c / X$ to $c^{\prime} / X$ in rank 2 .

- If $X \in L_{2}\left(c, c^{\prime}\right)$, then the hyperplanes of $\mathcal{A}_{X}$ can be crossed by $r$ in one of two possible linear orders, as in Figure 2.1

Consequently, given two minimal galleries $r, r^{\prime} \in \mathcal{R}\left(c, c^{\prime}\right)$, one can again speak of the separation set $L_{2}\left(r, r^{\prime}\right)$ as the subset of codimension-two subspaces $X$ in $L_{2}\left(c, c^{\prime}\right)$ on which $r, r^{\prime}$ disagree 5

\footnotetext{
${ }^{5}$ We apologize for the slightly confusing overuse of " $L_{2}$ " in our notation: we now have not only the set $L_{2}$, which is the collection of all codimension-two intersection subspaces of $\mathcal{A}$, but
} 
Definition 5.1 (The graph $G_{2}\left(c, c^{\prime}\right)$ ). Given the arrangement $\mathcal{A}$ and two chambers $c, c^{\prime} \in \mathcal{C}$, define a graph $G_{2}\left(c, c^{\prime}\right)$ whose vertex set is the set $\mathcal{R}\left(c, c^{\prime}\right)$ of minimal galleries $c$ to $c^{\prime}$, and having an edge between two galleries $\left\{r, r^{\prime}\right\}$ exactly when $\left|L_{2}\left(r, r^{\prime}\right)\right|=1$.

The previous discussion shows that the map $L_{2}: \mathcal{R}\left(c, c^{\prime}\right) \times \mathcal{R}\left(c, c^{\prime}\right) \longrightarrow 2^{L_{2}\left(c, c^{\prime}\right)}$ provides a set-valued metric on $G_{2}\left(c, c^{\prime}\right)$, taking values in $L_{2}\left(c, c^{\prime}\right)$. That discussion also shows that the generalization of the second assertion in Proposition 2.3 still holds: having fixed a base gallery $r_{0}$ in $\mathcal{R}\left(c, c^{\prime}\right)$, then any other gallery $r$ in $\mathcal{R}\left(c, c^{\prime}\right)$ is uniquely determined by its separation set $L_{2}\left(r_{0}, r\right)$. One also has this immediate consequence of Propositions 3.4 and 3.5 .

Corollary 5.2. The graph $G:=G_{2}\left(c, c^{\prime}\right)$ is always bipartite, and its distance function satisfies $d_{G}\left(r, r^{\prime}\right) \geq\left|L_{2}\left(r, r^{\prime}\right)\right|$.

Remark 5.3. Whenever $c_{0}, c, c^{\prime} \in \mathcal{C}$, satisfy $L_{1}\left(c_{0}, c\right) \subset L_{1}\left(c_{0}, c^{\prime}\right)$, one can define an injection $\mathcal{R}\left(c_{0}, c\right) \hookrightarrow \mathcal{R}\left(c_{0}, c^{\prime}\right)$ : fix any minimal gallery $r$ from $c$ to $c^{\prime}$, and then concatenation with $r$ as a suffix gives such an injection. It is easily seen that this leads to an embedding of the graph $G_{2}\left(c_{0}, c\right) \hookrightarrow G_{2}\left(c_{0}, c^{\prime}\right)$ as a vertex-induced subgraph.

In particular, although the graphs $G_{2}\left(c_{0}, c\right)$ with their set-valued metric do not in general have a $\mathbb{Z}_{2}$-equivariant involution, they are always vertex-induced subgraphs of the graph $G_{2}\left(c_{0},-c_{0}\right)$, which does have such an involution.

Remark 5.4. When $\mathcal{A}$ is the arrangement of reflecting hyperplanes for a finite real reflection group $W$, the (simply) transitive $W$-action on the chambers means that any graph $G_{2}\left(c, c^{\prime}\right)$ is isomorphic to the graph $G(w):=G_{2}\left(c_{0}, w\left(c_{0}\right)\right)$ for some fixed choice of a base chamber $c_{0}$ and some group element $w$ in $W$. It is not hard to check that this graph $G(w)$ is the graph of reduced words for $w$ discussed in the Introduction.

Furthermore, in this situation, the sets

$$
\begin{aligned}
& L_{1}(w):=L_{1}\left(c_{0}, w\left(c_{0}\right)\right), \\
& L_{2}(w):=L_{2}\left(c_{0}, w\left(c_{0}\right)\right)
\end{aligned}
$$

have the following reflection group interpretations:

- $L_{1}(w)$ is the usual (left-)inversion set of $w$, that is, the collection of positive roots $\alpha_{H}$ for $W$ which are sent to negative roots by $w^{-1}$.

- $L_{2}(w)$ is the collection of rank two subroot systems $\Phi_{X}$ having the property that $w^{-1}$ sends every positive root in $\Phi_{X}$ to a negative root.

One then has the following extension of Theorem 4.9 .

Theorem 5.5. Let $\mathcal{A}$ be a real (central, essential) hyperplane arrangement in $\mathbb{R}^{d}$ which is supersolvable, let $F:=\left\{X_{i}\right\}_{i=0}^{d}$ be a modular flag for $\mathcal{A}$, let $c_{0}$ be any chamber incident to $F$, and let $c$ be any other chamber.

also two functions called $L_{2}(-,-)$, namely

$$
\begin{aligned}
& \mathcal{C} \times \mathcal{C} \quad \longrightarrow 2^{L_{2}} \\
& \left(c \quad, \quad c^{\prime}\right) \quad \longmapsto L_{2}\left(c, c^{\prime}\right) \text {, } \\
& \mathcal{R}\left(c, c^{\prime}\right) \quad \times \mathcal{R}\left(c, c^{\prime}\right) \quad \longrightarrow \quad 2^{L_{2}\left(c, c^{\prime}\right)} \\
& \left(r \quad, \quad r^{\prime}\right) \quad \longmapsto L_{2}\left(r, r^{\prime}\right) .
\end{aligned}
$$

We hope that context resolves any confusion that arises within this section. 
(i) There is a unique minimal gallery $r_{0}$ from $c_{0}$ to $c$ incident to $F$.

(ii) This minimal gallery $r_{0}$ is an $L_{2}$-accessible vertex for the graph $G_{2}\left(c_{0}, c\right)$ on the galleries $\mathcal{R}$ from $c_{0}$ to $c$.

Proof. The proof of assertion (i) is by induction on $d$ exactly as in the proof of Theorem 4.9. This unique gallery $r_{0}$ is obtained by applying induction to the $(d-1)$-dimensional supersolvable arrrangement $\mathcal{A}_{\ell}$, lifting the unique gallery from $c_{0} / \ell$ to $c / \ell$ incident to $F / \ell$ in order to first cross all hyperplanes in $L_{1}\left(c_{0}, c\right) \cap \mathcal{A}_{\ell}$. One must complete it by then crossing the hyperplanes in $L_{1}\left(c_{0}, c\right) \backslash \mathcal{A}_{\ell}$ in the linear order which is the restriction of the one from Proposition 4.6 (i).

The proof of assertion (ii) is also by induction on $d$ exactly as in the proof of Theorem 4.9. One wishes to show that for any gallery $r \neq r_{0}$ from $c_{0}$ to $c$, there will be another gallery $r^{\prime}$ having $L_{2}\left(r_{0}, r^{\prime}\right) \subset L_{2}\left(r_{0}, r\right)$ and $\left|L_{2}\left(r, r^{\prime}\right)\right|=1$. Again there are two cases, depending upon whether (Case 1) or not (Case 2) the gallery $r$ crosses all the hyperplanes of $L_{1}\left(c_{0}, c\right) \cap \mathcal{A}_{\ell}$ before crossing any hyperplanes of $L_{1}\left(c_{0}, c\right) \backslash \mathcal{A}_{\ell}$. There is no essential change in the proof of Case 1.

In Case 2, one must note that the exhibited gallery $r^{\prime}$ having $L_{2}\left(r, r^{\prime}\right)=\{X\}$ satisfies in addition that $X$ lies in $L_{2}\left(c_{0}, c\right)$. This is immediate from the fact that $r$ crossed every hyperplane of $\mathcal{A}_{X}$ on its way from $c_{0}$ to $c$, so that $\mathcal{A}_{X} \subset L_{1}\left(c_{0}, c\right)$, that is, $X$ lies in $L_{2}\left(c_{0}, c\right)$.

Example 5.6. Continuing Example 4.10, for the reflection arrangement $\mathcal{A}$ of type $A_{5}$, choose as modular flag $F:=\left\{X_{i}\right\}_{i=0}^{5}$, where $X_{i}:=\left\{x_{1}=x_{2}=\cdots=x_{6-i}\right\}$. The chamber $c_{0}:=\left\{x_{1}<\cdots<x_{6}\right\}$ corresponding to the identity permutation is incident to the above modular flag $F$. Let $w=316425 \in \mathfrak{S}_{6}$. The unique gallery $r_{0}$ from $c_{0}$ to $c:=w\left(c_{0}\right)$ incident to $F$ crosses the hyperplanes in this order:

$$
\begin{aligned}
& H_{23}, H_{13}, \\
& H_{24}, \\
& H_{56}, H_{26}, H_{46} .
\end{aligned}
$$

The corresponding sequence of chambers in the gallery is indexed by the permutations

$$
123456 \stackrel{H_{23}}{-} 132456 \stackrel{H_{13}}{-} 312456 \stackrel{H_{24}}{-} 314256 \stackrel{H_{56}}{-} 314265 \stackrel{H_{26}}{-} 314625 \stackrel{H_{46}}{-} 316425,
$$

which corresponds to the following reduced decomposition for $w$

$$
\begin{aligned}
& \left(s_{2}, s_{1},\right. \\
& s_{3}, \\
& \left.s_{5}, s_{4}, s_{3}\right) .
\end{aligned}
$$

Corollary 5.7. Let $\mathcal{A}$ be a real (central, essential) hyperplane arrangement in $\mathbb{R}^{d}$ which is supersolvable, let $F:=\left\{X_{i}\right\}_{i=0}^{d}$ be a modular flag for $\mathcal{A}$, and let $c_{0}$ be any chamber incident to $F$. Let $c$ be any other chamber, and let $r_{0}$ be the unique minimal gallery from $c_{0}$ to $c$ incident to $F$.

Then in the graph $G:=G_{2}\left(c_{0}, c\right)$ of minimal galleries from $c_{0}$ to $c$, any two galleries $r, r^{\prime}$ satisfy

$$
\begin{aligned}
\left|L_{2}\left(r, r^{\prime}\right)\right| \leq d_{G}\left(r, r^{\prime}\right) & \leq\left|L_{2}\left(r_{0}, r\right)\right|+\left|L_{2}\left(r_{0}, r^{\prime}\right)\right| \\
& \leq 2\left|L_{2}\left(c_{0}, c\right)\right| .
\end{aligned}
$$

Thus the diameter of $G$ is at most $2\left|L_{2}\left(c_{0}, c\right)\right|$. 
In particular, when $\mathcal{A}$ is a reflection arrangement of type $A, B$ or dihedral type $I_{2}(m)$, so that $G=G(w)$ for some $w \in W$, any two reduced words $r, r^{\prime}$ satisfy

$$
\begin{aligned}
\left|L_{2}\left(r, r^{\prime}\right)\right| \leq d_{G}\left(r, r^{\prime}\right) & \leq\left|L_{2}\left(r_{0}, r\right)\right|+\left|L_{2}\left(r_{0}, r^{\prime}\right)\right| \\
& \leq 2\left|L_{2}(w)\right| .
\end{aligned}
$$

Thus the diameter of $G$ is at most $2\left|L_{2}(w)\right|$.

Example 5.8. Example 3.11 and the four words listed in (3) show that both the upper and lower bounds on $d_{G}\left(r, r^{\prime}\right)$ given in Corollary 5.7 need not be tight, even for $G=G\left(w_{0}\right)$ in type $A_{n-1}$.

In light of Theorem 1.1 one might wonder whether the upper bound of $2\left|L_{2}(w)\right|$ for the diameter of $G(w)$ in types $A, B$ can be improved to an upper bound of $\left|L_{2}(w)\right|$. However, we mention here some examples in types $A_{3}, B_{3}$ showing that even an upper bound of $\left|L_{2}(w)\right|$ for the diameter of $G(w)$ is not always tight.

In type $A_{3}$, where $W$ is the symmetric group $\mathfrak{S}_{4}$, the permutation $w=3412$ has two reduced words

$$
\begin{aligned}
\mathcal{R}(w)=\{ & \left(s_{2}, s_{3}, s_{1}, s_{2}\right), \\
& \left.\left(s_{2}, s_{1}, s_{3}, s_{2}\right)\right\}
\end{aligned}
$$

so that the graph $G(w)$ is a single edge, having diameter 1 . However, in this case, $L_{2}(w)=\left\{X_{13,24}, X_{14,23}\right\}$, so that $\left|L_{2}(w)\right|=2$.

One encounters a similar phenomenon in type $B_{3}$, for the signed permutation $w$ that maps the standard basis vectors $e_{1}, e_{2}, e_{3}$, respectively, to $-e_{3},-e_{2},-e_{1}$, respectively. This $w$ has only two reduced words

$$
\begin{aligned}
& \mathcal{R}(w)=\left\{\left(s_{0}, s_{1}, s_{0}, s_{2}, s_{1}, s_{0}\right),\right. \\
& \left.\left(s_{0}, s_{1}, s_{2}, s_{0}, s_{1}, s_{0}\right)\right\}
\end{aligned}
$$

with respect to the Coxeter generators $S=\left\{s_{0}, s_{1}, s_{2}\right\}$ from Example 4.11, so that the graph $G(w)$ is a single edge, having diameter 1 . But $L_{2}(w)$ consists of the three subspaces of codimension-two of the form $\left\{x_{i}=0, x_{j}+x_{k}=0\right\}$ with $\{i, j, k\}=\{1,2,3\}$, so that $\left|L_{2}(w)\right|=3$.

These examples suggest the following conjecture 6

Conjecture 5.9. For an element $w$ in the symmetric group $\mathfrak{S}_{n}$,

$$
\frac{1}{2}\left|L_{2}(w)\right| \leq \operatorname{diameter}(G(w)) \leq\left|L_{2}(w)\right| .
$$

For an element $w$ in the hyperoctahedral group $B_{n}$,

$$
\frac{1}{3}\left|L_{2}(w)\right| \leq \operatorname{diameter}(G(w)) \leq\left|L_{2}(w)\right| .
$$

Remark 5.10. The authors would like to thank an anonymous referee for pointing out the following connection between Theorems 4.9, 5.5, and the work of Armstrong [1] on sorting orders in Coxeter groups.

Let $W$ be a real reflection group $W$, with reflection arrangement $\mathcal{A}$, and assume $\mathcal{A}$ is supersolvable with a choice of modular flag $F$. Theorem 4.9 shows there is a unique minimal gallery $r_{0}$ from $c_{0}$ to $-c_{0}$ incident to $F$, which corresponds to a

\footnotetext{
${ }^{6}$ This conjecture has been checked only for the symmetric groups $S_{n}$ with $n \leq 5$, and for the hyperoctohedral groups $B_{n}$ with $n \leq 3$.
} 
particular reduced word $\mathbf{w}_{0}$ for the longest element $w_{0}$ in $W$. Theorem 5.5 then shows that for any other chamber $c$, there is again a unique minimal gallery $r$ from $c_{0}$ to $c$ incident to $F$. If $w$ is the unique element of $W$ for which $c=w\left(c_{0}\right)$, then this minimal gallery $r$ corresponds to a particular reduced word $\mathbf{w}$ for $w$.

Meanwhile in Armstrong's work, any choice of a reduced word $\mathbf{w}_{0}$ for $w_{0}$ induces, for each $w$ in $W$, a particular reduced word $\mathbf{w}$ for $w$, which he calls the $\mathbf{w}_{0}$-sorted word for $w$. Specifically, $\mathbf{w}$ is the lexicographically leftmost subword of $\mathbf{w}_{0}$ that gives a reduced word for $w$.

One can show that these two constructions are the same: the word w corresponding to the gallery $r$ incident to $F$ is the same as the $\mathbf{w}_{0}$-sorted word for $w$. This can be shown using an induction on the rank $d$, similar to the one employed in the proof of Theorems 4.9 and 5.5, together with the compatibility of parabolic coset factorization with weak Bruhat orders, and [1, Thm. 4.2].

\section{ACKNOWLEDGEMENTS}

The authors thank Anders Björner, Francesco Brenti, Patrick Dehornoy, Axel Hultman, Arkadius Kalka, Jim Lawrence, Thomas McConville, John Sullivan, and Günter M. Ziegler for helpful discussions and references. They thank Rob Edman for computations in types $D_{4}, F_{4}$ checking the non- $L_{2}$-accessibility of lexicographcially first reduced words. They thank Nathan Reading, Hugh Thomas, and an anonymous referee for corrections of typos and errors in an earlier version. Lastly, they thank another anonymous referee for helpful suggestions, including the content of Remark 5.10.

\section{REFERENCES}

[1] D. Armstrong, The sorting order on a Coxeter group. J. Combin. Theory Ser. A 116 (2009), 1285-1305. MR2568800 (2010m:20057)

[2] C.A. Athanasiadis and F. Santos, Monotone paths on zonotopes and oriented matroids. Canad. J. Math. 53 (2001), 1121-1140. MR1863845(2003a:52031)

[3] C.A. Athanasiadis, P.H. Edelman and V. Reiner, Monotone paths on polytopes. Math. Zeit. 235 (2000), 315-334. MR.1795510 (2002a:52011)

[4] M. Autord and P. Dehornoy, On the distance between the expressions of a permutation. arXiv:0902.3074. MR2673022

[5] H. Barcelo and E. Ihrig, Lattices of parabolic subgroups in connection with hyperplane arrangements. J. Algebraic Combin. 9 (1999), no. 1, 5-24. MR.1676736 (2000g:52023)

[6] H.J. Baues, Geometry of loop spaces and the cobar construction. Mem. Amer. Math. Soc. 25, Amer. Math. Soc., Providence, RI, 1980. MR:567799 (81m:55010)

[7] L.J. Billera, M.M. Kapranov and B. Sturmfels, Cellular strings on polytopes. Proc. Amer. Math. Soc. 122 (1994), 549-555. MR1205482 (95a:52020)

[8] L.J. Billera and B. Sturmfels, Fiber polytopes, Ann. of Math. (2) 135 (1992), 527-549. MR.1166643 (93e:52019)

[9] A. Björner, Essential chains and homotopy type of posets. Proc. Amer. Math. Soc. 116 (1992), 1179-1181. MR1140664 (94b:06001)

[10] A. Björner and F. Brenti, Combinatorics of Coxeter groups. Graduate Texts in Mathematics, 231. Springer, New York, 2005. MR2133266 (2006d:05001)

[11] A. Björner, P.H. Edelman, and G.M. Ziegler, Hyperplane arrangements with a lattice of regions. Discrete Comput. Geom. 5 (1990), 263-288. MR.1036875 (90k:51036)

[12] A. Björner, M. Las Vergnas, B. Sturmfels, N. White, and G.M. Ziegler, Oriented matroids. Encyclopedia of Mathematics and Its Applications 46. Cambridge University Press, Cambridge, 1993. MR:1226888 (95e:52023)

[13] R. Cordovil, Sur les matrö̈des orientés de rang 3 et les arrangements de pseudodroites dans le plan projectif réel. Europ. J. Combin. 3 (1982), 307-318. MR687729 (84j:05031) 
[14] R. Cordovil and M.L. Moreira, A homotopy theorem on oriented matroids. Discrete Math. 111 (1993), 131-136. MR 1210090 (94d:52016)

[15] P. Deligne, Les immeubles des groupes de tresses généralisés. Invent. Math. 17 (1972), 273302. MR0422673(54:10659)

[16] P.H. Edelman, A partial order on the regions of $\mathbb{R}^{n}$ dissected by hyperplanes. Trans. Amer. Math. Soc. 283 (1984), 617-631. MR737888(85k:51030)

[17] S. Felsner and H. Weil, A theorem on higher Bruhat orders. Discrete Comput. Geom. 23 (2000), 121-127. MR 1727126 (2001e:52044)

[18] J.E. Humphreys, Reflection groups and Coxeter groups. Cambridge Studies in Advanced Mathematics. 29. Cambridge University Press, Cambridge, 1990. MR:1066460 (92h:20002)

[19] Yu.I. Manin and V.V. Schechtman, Arrangements of hyperplanes, higher braid groups and higher Bruhat orders. Algebraic number theory, 289-308, Adv. Stud. Pure Math. 17, Academic Press, Boston, MA, 1989. MR1097620 (91m:32042)

[20] P. Orlik and H. Terao, Arrangements of hyperplanes. Grundlehren der Mathematischen Wissenschaften 300. Springer-Verlag, Berlin, 1992 MR1217488 (94e:52014)

[21] J. Rambau, A suspension lemma for bounded posets. J. Combin. Theory Ser. A 80 (1997), 374-379. MR1485152 (99a:06005)

[22] V. Reiner, The generalized Baues problem. New perspectives in algebraic combinatorics (Berkeley, CA, 1996-97), 293-336, Math. Sci. Res. Inst. Publ. 38, Cambridge Univ. Press, Cambridge, 1999. MR1731820(2000i:52023)

[23] V. Reiner, Note on the expected number of Yang-Baxter moves applicable to reduced decompositions. European J. Combin. 26 (2005), 1019-1021. MR2143207 (2005m:05236)

[24] J. Richter-Gebert, Oriented matroids with few mutations, Discrete and Computational Geometry 10 (1993), 251-269. MR:1226979(94f:52019)

[25] M. Salvetti, Topology of the complement of real hyperplanes in $\mathbb{C}^{n}$. Invent. Math. 88 (1987), 603-618. MR884802 (88k:32038)

[26] B. Shapiro, M. Shapiro and A. Vainshtein, Connected components in the intersection of two open opposite Schubert cells in $\mathrm{SL}_{n}(R) / B$. Internat. Math. Res. Notices 1997, no. 10, 469-493. MR1446839 (98e:14054)

[27] R.P. Stanley, Supersolvable lattices. Algebra Universalis 2 (1972), 197-217. MR0309815 (46:8920)

[28] R.P. Stanley, On the number of reduced decompositions of elements of Coxeter groups. European J. Combin. 5 (1984), 359-372. MR.782057 (86i:05011)

[29] R.P. Stanley, An introduction to hyperplane arrangements. Geometric combinatorics, 389496, IAS/Park City Math. Ser. 13, Amer. Math. Soc., Providence, RI, 2007. MR2383131

[30] J.A. Tits, Le problème des mots dans les groupes de Coxeter. Symposia Mathematica (INDAM, Rome, 1967/68), 1(1969), 175-185 Academic Press, London. MR0254129 (40:7339)

[31] J.A. Tits, A local approach to buildings. The geometric vein, pp. 519-547, Springer, New York-Berlin, 1981.

[32] G.M. Ziegler, Higher Bruhat orders and cyclic hyperplane arrangements. Topology 32 (1993), no. 2, 259-279. MR1217068 (94e:52015)

[33] G.M. Ziegler, Lectures on polytopes. Graduate Texts in Mathematics 152. Springer-Verlag, New York, 1995. MR.1311028 (96a:52011)

School of Mathematics, University of Minnesota, Minneapolis, Minnesota 55455

E-mail address: reiner@math.umn.edu

Department of Mathematics, Bar-Ilan University, 52900 Ramat-Gan, Israel

E-mail address: yuvalr@math.biu.ac.il 